\title{
Magnetic Resonance Imaging of Trunk Musculature and Intervertebral Discs in Patients with Spinal Cord Injury with Thoracolumbar Vertebral Fractures: A Prospective Study
}

\author{
Roop Singh ${ }^{1}$, Jitendra Wadhwani ${ }^{1}$, Gaurav Punia ${ }^{1}$, Rajesh Kumar Rohilla ${ }^{1}$, Kiranpreet Kaur ${ }^{2}$ \\ ${ }^{I}$ Department of Orthopaedic Surgery, Paraplegia \& Rehabilitation, Pandit Bhagwat Dayal Sharma Post Graduate Institute of Medical Sciences, Rohtak, India \\ ${ }^{2}$ Department of Anaesthesiology and Critical Care, Pandit Bhagwat Dayal Sharma Post Graduate Institute of Medical Sciences, Rohtak, India
}

Study Design: This study is a prospective clinical study.

Purpose: This study aims to evaluate the characteristics of trunk musculature and intervertebral discs by using magnetic resonance imaging in patients with spinal cord injuries (SCIs) with thoracic and lumbar fractures.

Overview of Literature: Muscle atrophy is an immediate consequence of $\mathrm{SCl}$ and is associated with secondary complications. At present, there are limited clinical data on muscle and disc responses to fractures of the thoracic and lumbar spine.

Methods: A total of 51 patients with a mean age of $31.75 \pm 10.42$ years who suffered traumatic SCI were included in this study. Complete neurological examinations (American Spinal Injury Association grading) and magnetic resonance imaging (MRI) were performed at the time of admission and at 3-6 months after injury to study the neurological status and disc and trunk parameters. The type of management (operative or conservative) was decided on the basis of clinical, radiological, and MRI evaluations, and a robust rehabilitation program was initiated.

Results: Disc parameters including disc angle, skin angle, cross-sectional area (CSA), and disc height and trunk parameters (mean trunk width, mean trunk depth, and CSA of the lumbar muscles) decreased significantly $(p<0.001)$ during the first 3 months after SCI. However, improvements were observed in disc and muscle parameters at the 6-month follow-up, but these parameters did not return to normal levels. Neither initial neurological status (complete vs. incomplete) nor type of management (operative vs. conservative) had a significant effect on these parameters.

Conclusions: Spinal trauma leads to alterations in the morphology of the vertebral column, spinal cord, intervertebral discs, and paraspinal muscles in the initial phase of injury. The extent of these changes may determine the initial neurological deficit and subsequent recovery. Although this study did not identify any statistically significant effect of neurological status or management strategy on these parameters, rehabilitation was found to result in the improvement of these parameters in the later phase of recovery. Future studies are required to evaluate the exact causes of these alterations and the potential benefits of rehabilitation strategies and to minimize these changes.

Keywords: Spinal fractures; Muscles; Magnetic resonance imaging; Spinal cord injuries; Intervertebral disc; Rehabilitation

Received Jan 6, 2019; Revised Sep 25, 2019; Accepted Oct 23, 2019

Corresponding author: Roop Singh

Department of Orthopaedic Surgery, 52/9-J, Medical Enclave, Post Graduate Institute of Medical Sciences, Rohtak-124001, Haryana, India

Tel: +91-1262-283171, Fax: +91-1262-281308, E-mail: drroopsingh@rediffmail.com 


\section{Introduction}

Acute traumatic spinal cord injury (SCI) is one of the most devastating injuries for the human body. This injury causes immediate and, in some areas, permanent gravitational unloading, thus resulting in structural changes due to disuse and associated metabolic consequences. Osteoporosis and muscle atrophy are the frequently encountered complications of SCI $[1,2]$, and these conditions occur rapidly after injury and are associated with several secondary complications. Inactivation and extreme unloading following SCI can lead to marked atrophy of the leg and thigh skeletal muscles within a few months of injury [3].

A number of studies have evaluated the utility of various magnetic resonance imaging (MRI) parameters in relation to neurological performance following SCI; however, there is little information available on the response of intervertebral disc and paravertebral muscles to spinal trauma. The present study was conducted to evaluate the MRI data of trunk musculature and discs in patients who had suffered SCI with thoracic and lumbar vertebral fractures. We hypothesized that spinal trauma affects the trunk musculature and intervertebral discs and suggest that the information presented here deepens our understanding of muscular wasting, appropriate rehabilitation strategies, and approaches for improving the ultimate outcome of SCI.

\section{Materials and Methods}

This prospective study was performed from June 2015 to December 2017 and included patients who presented to our tertiary care institution with posttraumatic SCI. Owing to time constraints and the paucity of previous data related to the present research question, this study was conducted as a pilot study with 51 subjects recruited prospectively on the basis of the availability of patients undergoing treatment. The study and all its protocols were approved by the institutional review board and ethical committee (IRB approval no., Endst. No. Surg/ Dean/16/1640-45; dated 08/06/2016). Written informed consent was obtained from all individual participants. We recruited all adult patients who suffered posttraumatic SCI. After emergency stabilization, a detailed history was taken from each patient in chronological order, and a thorough general physical and neurological examination was performed.

Each patient underwent a thorough clinical examination including spinal X-ray and MRI. The 1.5 Tesla machine was used to obtain MRI data with the patient lying comfortably in the supine position with knees and hips extended. Multislice sagittal and transverse sections were taken with MRI sequences. The locations of vertebral levels for MRI were determined from pilot parasagittal sections. Measurements were performed using on-screen calipers. Various parameters (detailed in Table 1) were measured at the L1-L2, L2-L3, L3-L4, L4-L5, and L5S1 level of the intervertebral disc (as per the institutional protocol published previously [4]).

The clinical assessments of sensory score, motor score, and zone of partial preservation were performed at the time of admission and at 3 days, 7 days, 3 months, and 6 months postadmission according to the international scoring system of the American Spinal Injury Association (ASIA) [5]. Deep anal pressure (DAP), voluntary anal contraction (VAC), and any anal sensations were noted during each clinical assessment. The neurological level of injury (sensory and motor) was evaluated and defined as complete or incomplete. SCI was classified into five categories (A to E) according to the ASIA impairment scale (AIS) [6].

Plain roentgenogram examinations (lateral images, anteroposterior film) were conducted. Routine laboratory investigations including hemoglobin level, bleeding

Table 1. Various quantitative parameters measured with section at level of intervertebral discs

\begin{tabular}{|c|c|c|}
\hline \multicolumn{2}{|r|}{ Variable } & Description \\
\hline 1 & Trunk dimensions & Width (maximum width was be taken) \\
\hline & & $\begin{array}{l}\text { Depth (anteroposterior diameter of trunk at mid- } \\
\text { sagittal section) }\end{array}$ \\
\hline \multirow[t]{6}{*}{2} & CSA of muscle & Erector spinae \\
\hline & & Multifidus \\
\hline & & Psoas major \\
\hline & & Quadratus lumborum \\
\hline & & Rectus abdominis \\
\hline & & Obliques \\
\hline 3 & Disc angle & $\begin{array}{l}\text { The angle between the vertical line and the midplane } \\
\text { of each lumbar disc }\end{array}$ \\
\hline 4 & Skin angle & $\begin{array}{l}\text { The angle between the vertical line and the line tan- } \\
\text { gent to the overlying skin }\end{array}$ \\
\hline 5 & CSA of disc & \\
\hline
\end{tabular}

CSA, cross-sectional area. 
time, clotting time, complete urine examination, blood urea, blood sugar, serum electrolytes, electrocardiogram, and chest roentgenogram were performed in all cases. We performed MRI within 48 hours of injury in all cases. Patients who required surgery for unstable vertebral column injuries were operated on according to the requirements. Spinal stability was evaluated for thoracolumbar injuries according to lumbar spinal stenosis [7], and the thoracolumbar injury classification system [8]. Unstable spine was considered an indication for spinal surgery (either stabilization alone or stabilization with decompression). The type of surgery was decided on the basis of the characteristics of the fracture. Patients with stable spinal injuries were advised to stay on bed rest until pain subsided and were later mobilized with the use of braces. Patients who underwent surgical stabilization were usually mobilized on the third day after surgery following dressing change unless there were any contraindications. Rehabilitation for early mobilization and active/assisted limb and paraspinal muscle strengthening exercises were initiated as soon as possible for all patients.

Patients attended follow-up appointments at 3 and 6 months postinjury. Clinical evaluations and plain radiography examinations were performed at each follow-up, and MRI was performed at the 3- and 6-month follow-up appointments. Neurological recovery was documented on the basis of AIS.

The collected data were compiled and entered into spreadsheets. Statistical analysis was performed using the statistical software IBM SPSS for Windows ver. 20.0 (IBM Corp., Armonk, NY, USA). Continuous variables are presented as mean and standard deviation, and categorical variables are presented as proportions. The independent Student $t$-test was used to analyze statistical differences in continuous variables between two independent groups. To compare more than two independent groups or ordinal dependent variables, we used one-way analysis of variance (ANOVA). For repeated-measures analysis, repeatedmeasures ANOVA, Friedman's ANOVA, and Cochran's Q test were used on the basis of whether the dependent variable being measured was continuous, ordinal, or categorical, respectively. The level of significance was taken as 5\% with $95 \%$ confidence intervals.

\section{Results}

Fifty-one patients (40 males, 11 females) were included in this study. The mean age of the study population was $31.75 \pm 10.42$ years (range, $18-65$ years), and the modal group was $21-30$ years $(\mathrm{n}=27,52.9 \%)$.

Table 2 shows the symptomatology at admission and subsequent evaluations. All symptoms decreased significantly over time $(p<0.001)$, except in two patients wherein incontinence persisted at 6 months postadmission.

Table 3 shows the neurological involvement and improvement over time. Neurological involvement showed a statistically significant improvement $(p<0.001)$.

There was no significant difference in the distribution of initial clinical assessment values (VAC, motor index score, and sensory index score), and DAP was managed operatively and conservatively. The superficial abdominal reflex was initially absent in $45.1 \%$ (23) of patients but remained absent in only $31.4 \%$ (16) of patients at the 6 -month follow-up ( $p=0.001$ ). The Babinski reflex was initially absent and remained absent throughout the followup for all patients. Knee and ankle reflexes were initially absent in $92.2 \%$ (47) of patients but remained absent in only $25.5 \%$ (13) of patients at the 6 -month follow-up; this

Table 2. Distribution of subjects according to their symptomatology ( $n=51)$

\begin{tabular}{|c|c|c|c|c|}
\hline Symptoms & Initial & $3 \mathrm{mo}$ & $6 \mathrm{mo}$ & $p$-value \\
\hline Pain & & & & $<0.001^{\text {a) }}$ \\
\hline Absent & 0 & $34(66.7)$ & $49(96.1)$ & \\
\hline Mild & 0 & $17(33.3)$ & $2(3.9)$ & \\
\hline Moderate & 0 & 0 & 0 & \\
\hline Severe & $51(100.0)$ & 0 & 0 & \\
\hline Swelling & & & & $<0.001^{a)}$ \\
\hline Absent & 0 & $48(94.1)$ & $51(100.0)$ & \\
\hline Mild & 0 & $3(5.9)$ & 0 & \\
\hline Moderate & $20(39.2)$ & 0 & 0 & \\
\hline Severe & $31(60.8)$ & 0 & 0 & \\
\hline Deformity & & & & $<0.001^{\text {a) }}$ \\
\hline Absent & 0 & $33(64.7)$ & $46(90.2)$ & \\
\hline Mild & $2(3.9)$ & $18(35.3)$ & $5(9.8)$ & \\
\hline Moderate & $38(74.5)$ & 0 & 0 & \\
\hline Severe & $11(21.6)$ & 0 & 0 & \\
\hline Weakness upper limb & 0 & 0 & 0 & - \\
\hline Weakness lower limb & $49(96.1)$ & 18 (35.3) & $12(23.5)$ & $<0.001^{\text {b) }}$ \\
\hline Incontinence & 0 & 0 & 2 (3.9) & $0.135^{\mathrm{bl}}$ \\
\hline Retention & $46(90.2)$ & $16(31.4)$ & $10(19.6)$ & $<0.001^{b /}$ \\
\hline
\end{tabular}

Values are presented as number (\%).

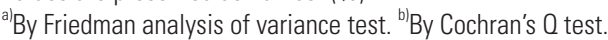


decrease was statistically significant $(p<0.001)$. The median neurological grade at initial presentation was $\mathrm{D}$. The grade improved to $\mathrm{E}$ in the first 3 months after admission and remained as $E$ for the next 3 months. Neurological recovery was found to be highly significant $(p<0.001)$ by the Friedman ANOVA test. Initially, more than half of the study population (56.9\%, 29 patients) were classified as grade D, 19.6\% (10) of patients were classified as grade A (complete injury), 15.7\% (8) of patients were classified as grade $\mathrm{C}$, and $3.9 \%$ (2) of patients were classified as grades $\mathrm{B}$ and $\mathrm{E}$ each. At the 6-month follow-up, the majority of patients exhibited no deficit (74.5\%, 38 patients), whereas $19.6 \%$ (10) were classified as grade C. Thirty-five (68.6\%) patients were managed conservatively, and 16 patients (31.37\%) were managed operatively. There was no significant difference in the distribution of the initial ASIA score between these two groups.

The mean trunk widths at various levels of the spinal cord decreased at the 3-month follow-up compared with that at the initial recordings but increased at the 6-month follow-up. Posthoc analysis revealed that this change is statistically significant $(p<0.001)$ at all disc levels of the lumbar spinal cord from L1-S1 and at all time points (Table 4).

Table 5 shows the mean trunk depths at various levels of the spinal cord. Except at the L2-L3 disc levels, mean trunk depth at all levels decreased at the 3-month followup compared with that at the initial recordings. However, the mean trunk depth increased between the 3- and 6-month follow-up. Posthoc analysis revealed that this change is statistically significant $(p<0.001)$ at all disc levels and at all time points, except for L3-L4 ( $p=0.049$, initial versus 3 months) and L4-L5 ( $p=1.000$, 3- versus 6-month

Table 3. Distribution of subjects according to their neurological assessment $(n=51)$

\begin{tabular}{|c|c|c|c|c|}
\hline Neurological assessment & Initial & $3 \mathrm{mo}$ & $6 \mathrm{mo}$ & $p$-value \\
\hline Decreased muscle tone & $49(96.1)$ & $18(35.3)$ & $13(25.5)$ & $<0.001^{\text {a) }}$ \\
\hline Mean MIS-upper limb & 50 & 50 & 50 & - \\
\hline Mean MIS-lower limb & $27.28 \pm 17.30$ & $40.51 \pm 14.96$ & $44.33 \pm 10.88$ & $<0.001^{\text {b) }}$ \\
\hline Voluntary anal contraction (absent) & $26(51.0)$ & $18(35.3)$ & $10(19.6)$ & $<0.001^{\text {a) }}$ \\
\hline SIS-light touch & $96.0 \pm 23.33$ & $105.84 \pm 12.58$ & $109.18 \pm 6.91$ & $<0.001^{\text {b) }}$ \\
\hline SIS-pin prick & $95.69 \pm 23.79$ & $105.10 \pm 14.96$ & $109.49 \pm 6.23$ & $<0.001^{\text {b) }}$ \\
\hline Temperature sense (absent) & $11(21.6)$ & $4(7.8)$ & $4(7.8)$ & $0.001^{\text {a) }}$ \\
\hline Deep anal pressure (absent) & $17(33.3)$ & $11(21.6)$ & $5(9.8)$ & $<0.001^{\text {a) }}$ \\
\hline Clonus (absent) & $51(100.0)$ & $51(100.0)$ & $51(100.0)$ & $1.000^{\mathrm{a})}$ \\
\hline Zone of partial preservation & 0 & 0 & 0 & - \\
\hline Bladder-bowel involvement & $49(96.1)$ & $16(31.4)$ & $11(21.6)$ & $<0.001^{\text {a) }}$ \\
\hline
\end{tabular}

Values are presented as number (\%) or mean \pm standard deviation.

MIS, Motor Index Score; SIS, Sensory Index Score.

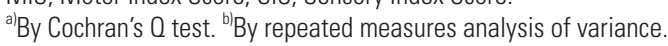

Table 4. Trunk width at various levels of lumbar discs initially and on follow-up ( $n=51)$

\begin{tabular}{|c|c|c|c|c|c|c|}
\hline \multirow{2}{*}{ Vertebral level } & \multicolumn{3}{|c|}{ Trunk width (mm) } & \multicolumn{3}{|c|}{ Intergroup comparisons: $p$-value ${ }^{\text {a) }}$ (post hoc analysis) } \\
\hline & Initial (A) & 3 mo (B) & $6 \mathrm{mo}(\mathrm{C})$ & $A-B$ & $A-C$ & $B-C$ \\
\hline L1-2 & $297.06 \pm 13.35$ & $282.25 \pm 13.39$ & $290.06 \pm 14.08$ & 0.000 & 0.000 & 0.000 \\
\hline L2-3 & $299.33 \pm 11.21$ & $287.55 \pm 11.15$ & $294.31 \pm 12.14$ & 0.000 & 0.000 & 0.000 \\
\hline L3-4 & $303.24 \pm 10.79$ & $291.43 \pm 11.05$ & $297.31 \pm 11.65$ & 0.000 & 0.000 & 0.000 \\
\hline$\lfloor 4-5$ & $305.16 \pm 9.17$ & $291.55 \pm 8.08$ & $299.78 \pm 9.49$ & 0.000 & 0.000 & 0.000 \\
\hline L5-S1 & $306.04 \pm 8.97$ & $292.27 \pm 13.76$ & $299.53 \pm 11.07$ & 0.000 & 0.000 & 0.000 \\
\hline
\end{tabular}

Values are presented as mean \pm standard deviation. A-B means initial versus 3 months; $B-C$ means 3 months versus 6 months; A-C means initial versus 6 months. a) By repeated measures analysis of variance test. 
follow-up data).

The mean disc angle at all disc levels of the vertebral column (except for L2-L3 and L4-L5) was significantly decreased $(p<0.001)$ at the 6 -month follow-up. At the L2-L3 and L4-L5 levels, the mean disc angle was significantly decreased at the 3-month follow-up compared with the initial recordings but significantly increased at the 6-month follow-up $(p<0.001)$ (Table 6). Posthoc analysis showed that this change is not statistically significant at the L2-L3, L3-L4, or L4-L5 at the 3-month follow-up versus the 6-month follow-up.

The mean skin angle was decreased at follow-up compared with the initial values at all lumbar vertebral levels. Posthoc analysis showed that this change was not statistically significant at the L2-L3 ( $p=0.194)$ or L4-L5 lumbar spine levels at the 3-month follow-up compared with the 6-month follow-up (Table 7).

Table 8 shows the mean cross-sectional area (CSA) of the disc at various levels of the lumbar spine at the time of admission and at follow-up. Posthoc analysis showed that

Table 5. Trunk depth at various levels of lumbar discs initially and on follow-up ( $n=51)$

\begin{tabular}{|c|c|c|c|c|c|c|}
\hline \multirow{2}{*}{ Vertebral level } & \multicolumn{3}{|c|}{ Trunk depth (mm) } & \multicolumn{3}{|c|}{ Intergroup comparisons: $p$-value ${ }^{\text {a) }}$ (post hoc analysis) } \\
\hline & Initial (A) & 3 mo (B) & $6 \mathrm{mo}(\mathrm{C})$ & $A-B$ & $A-C$ & $B-C$ \\
\hline L1-2 & $198.47 \pm 16.92$ & $176.65 \pm 12.00$ & $195.55 \pm 16.25$ & 0.000 & 0.019 & 0.000 \\
\hline L2-3 & $195.61 \pm 10.17$ & $190.63 \pm 13.04$ & $178.29 \pm 8.03$ & 0.002 & 0.000 & 0.000 \\
\hline Ł3-4 & $198.71 \pm 16.54$ & $179.18 \pm 13.58$ & $196.18 \pm 15.29$ & 0.000 & 0.049 & 0.000 \\
\hline$\lfloor 4-5$ & $202.55 \pm 15.48$ & $189.84 \pm 19.75$ & $193.98 \pm 16.17$ & 0.016 & 0.000 & 1.00 \\
\hline L5-S1 & $197.41 \pm 16.79$ & $185.76 \pm 15.95$ & $191.88 \pm 15.55$ & 0.000 & 0.000 & 0.000 \\
\hline
\end{tabular}

Values are presented as mean \pm standard deviation. A-B means initial versus 3 months; B-C means 3 months versus 6 months; $A-C$ means initial versus 6 months. a) By repeated measures analysis of variance test.

Table 6. Disc angle at various levels of lumbar discs initially and on follow-up ( $n=51)$

\begin{tabular}{|c|c|c|c|c|c|c|}
\hline \multirow{2}{*}{ Vertebral level } & \multicolumn{3}{|c|}{ Disc angle $\left({ }^{\circ}\right)$} & \multicolumn{3}{|c|}{ Intergroup comparisons: $p$-value ${ }^{\text {a) }}$ (post hoc analysis) } \\
\hline & Initial (A) & 3 mo (B) & $6 \mathrm{mo}(\mathrm{C})$ & $A-B$ & A-C & $B-C$ \\
\hline L1-2 & $6.98 \pm 0.81$ & $6.27 \pm 0.53$ & $6.02 \pm 0.86$ & 0.000 & 0.000 & 0.003 \\
\hline L2-3 & $6.76 \pm 0.79$ & $5.98 \pm 0.93$ & $6.12 \pm 1.01$ & 0.000 & 0.000 & 0.579 \\
\hline L3-4 & $1.75 \pm 0.44$ & $1.02 \pm 0.86$ & $0.84 \pm 0.81$ & 0.000 & 0.000 & 0.870 \\
\hline$\lfloor 4-5$ & $-6.88 \pm 0.86$ & $-6.06 \pm 0.86$ & $-6.18 \pm 1.05$ & 0.000 & 0.000 & 0.729 \\
\hline L5-S1 & $-21.35 \pm 2.90$ & $-19.37 \pm 2.91$ & $-20.00 \pm 3.30$ & 0.000 & 0.000 & 0.000 \\
\hline
\end{tabular}

Values are presented as mean \pm standard deviation. A-B means initial versus 3 months; B-C means 3 months versus 6 months; A-C means initial versus 6 months. a) By repeated measures analysis of variance test.

Table 7. Skin angle at various levels of lumbar spine initially and on follow-up ( $n=51)$

\begin{tabular}{|c|c|c|c|c|c|c|}
\hline \multirow{2}{*}{ Vertebral level } & \multicolumn{3}{|c|}{ Skin angle $\left({ }^{\circ}\right)$} & \multicolumn{3}{|c|}{ Intergroup comparisons: $p$-value ${ }^{\text {a) }}$ (post hoc analysis) } \\
\hline & Initial $(A)$ & $3 \mathrm{mo}(\mathrm{B})$ & $6 \mathrm{mo}(\mathrm{C})$ & $A-B$ & $A-C$ & $B-C$ \\
\hline L1-2 & $-96.02 \pm 3.04$ & $-93.02 \pm 4.53$ & $-93.41 \pm 3.83$ & 0.000 & 0.000 & 0.009 \\
\hline L2-3 & $-99.67 \pm 3.90$ & $-97.00 \pm 4.36$ & $-97.33 \pm 3.72$ & 0.000 & 0.000 & 0.194 \\
\hline Ł3-4 & $-105.33 \pm 1.26$ & $-103.00 \pm 1.43$ & $-104.00 \pm 1.65$ & 0.000 & 0.000 & 0.000 \\
\hline$\lfloor 4-5$ & $-115.67 \pm 2.52$ & $-111.00 \pm 2.97$ & $-113.00 \pm 2.97$ & 0.000 & 0.000 & NA \\
\hline L5-S1 & $-112.00 \pm 2.47$ & $-108.67 \pm 0.95$ & $-110.00 \pm 1.43$ & 0.000 & 0.000 & 0.000 \\
\hline
\end{tabular}

Values are presented as mean \pm standard deviation. A-B means initial versus 3 months; B-C means 3 months versus 6 months; A-C means initial versus 6 months. a) By repeated measures analysis of variance test. 
this change was not statistically significant at the L3-L4 ( $p=0.275,3$ - versus 6 -month follow-up), L4-L5 ( $p=0.492$, initial versus 3-month follow-up), or L5-S1 levels ( $p=1.000$, initial versus three-month follow-up; $p=0.075$, initial versus 6-month follow-up). There was no significant change in mean muscle disc ratio at any disc level, except L5-S1 ( $p=0.000$, initial versus 3 -month follow-up; $p=0.011,3$ - versus 6-month follow-up) (Table 9).

The mean intervertebral disc height was significantly decreased at the 3-month follow-up compared with the initial recordings at all levels, but this value was increased at the 6-month follow-up. Posthoc analysis showed that this change was not statistically significant at the L1-L2 ( $p=1.000$, initial versus 3 -month follow-up) or L4-L5 levels ( $p=0.151,3$ - versus 6-month follow-up) (Table 10).

Table 11 shows the mean CSA of lumbar muscles at all disc levels of the lumbar spine at the time of admission and follow-up. Intergroup comparisons were performed to investigate the changes over different periods of time. The results revealed a significant decrease in the mean

Table 8. Mean cross-sectional area of disc at various levels of lumbar spine initially and on follow-up ( $n=51$ )

\begin{tabular}{|c|c|c|c|c|c|c|}
\hline \multirow{2}{*}{ Vertebral level } & \multicolumn{3}{|c|}{ Cross-sectional area $\left(\mathrm{cm}^{2}\right)$} & \multicolumn{3}{|c|}{ Intergroup comparisons: $p$-value ${ }^{\text {a) }}$ (post hoc analysis) } \\
\hline & Initial (A) & 3 mo (B) & $6 \mathrm{mo}(\mathrm{C})$ & $A-B$ & $A-C$ & $B-C$ \\
\hline L1-2 & $14.82 \pm 0.61$ & $14.50 \pm 0.58$ & $14.67 \pm 0.58$ & 0.000 & 0.005 & 0.000 \\
\hline L2-3 & $14.85 \pm 0.59$ & $14.53 \pm 0.26$ & $14.60 \pm 0.22$ & 0.000 & 0.001 & 0.000 \\
\hline L3-4 & $14.89 \pm 0.57$ & $14.70 \pm 0.30$ & $14.73 \pm 0.35$ & 0.003 & 0.010 & 0.275 \\
\hline L4-5 & $14.88 \pm 0.58$ & $14.69 \pm 0.21$ & $14.78 \pm 0.18$ & 0.048 & 0.492 & 0.000 \\
\hline L5-S1 & $14.80 \pm 0.61$ & $14.80 \pm 0.38$ & $14.90 \pm 0.52$ & 1.000 & 0.075 & 0.000 \\
\hline
\end{tabular}

Values are presented as mean \pm standard deviation. A-B means initial versus 3 months; $B-C$ means 3 months versus 6 months; $A-C$ means initial versus 6 months. a) By repeated measures analysis of variance test.

Table 9. Muscle disc ratio at various levels of lumbar spine initially and on follow-up $(n=51)$

\begin{tabular}{|c|c|c|c|c|c|c|}
\hline \multirow{2}{*}{ Vertebral level } & \multicolumn{3}{|c|}{ Muscle disc ratio $\left(\mathrm{cm}^{2}\right)$} & \multicolumn{3}{|c|}{ Intergroup comparisons: $p$-value ${ }^{\text {a) }}$ (post hoc analysis) } \\
\hline & Initial (A) & $3 \mathrm{mo}(\mathrm{B})$ & $6 \mathrm{mo}(\mathrm{C})$ & $A-B$ & $A-C$ & $\mathrm{~B}-\mathrm{C}$ \\
\hline L1-2 & $0.55 \pm 0.05$ & $0.56 \pm 0.05$ & $0.54 \pm 0.05$ & 1.000 & 1.000 & 0.491 \\
\hline $\mathrm{L} 2-3$ & $0.56 \pm 0.05$ & $0.56 \pm 0.05$ & $0.54 \pm 0.05$ & 1.000 & 1.000 & 0.491 \\
\hline L3-4 & $0.55 \pm 0.05$ & $0.54 \pm 0.05$ & $0.54 \pm 0.05$ & 1.000 & 1.000 & 0.491 \\
\hline$\lfloor 4-5$ & $1.07 \pm 0.05$ & $1.05 \pm 0.05$ & $1.05 \pm 0.05$ & 0.032 & 0.694 & 1.000 \\
\hline L5-S1 & $1.35 \pm 0.07$ & $1.31 \pm 0.04$ & $1.34 \pm 0.05$ & 0.000 & 1.000 & 0.011 \\
\hline
\end{tabular}

Values are presented as mean \pm standard deviation. A-B means initial versus 3 months; $B-C$ means 3 months versus 6 months; A-C means initial versus 6 months. a) By repeated measures analysis of variance test.

Table 10. Intervertebral disc height at various levels of lumbar spine initially and on follow-up (n=51)

\begin{tabular}{|c|c|c|c|c|c|c|}
\hline \multirow{2}{*}{ Vertebral level } & \multicolumn{3}{|c|}{ Intervertebral disc height (mm) } & \multicolumn{3}{|c|}{ Intergroup comparisons: $p$-value ${ }^{\text {a) }}$ (post hoc analysis) } \\
\hline & Initial (A) & 3 mo (B) & $6 \mathrm{mo}(\mathrm{C})$ & $A-B$ & $A-C$ & $B-C$ \\
\hline L1-2 & $11.87 \pm 0.42$ & $11.73 \pm 0.45$ & $11.73 \pm 0.50$ & 0.000 & 0.000 & 1.000 \\
\hline L2-3 & $11.37 \pm 0.42$ & $11.23 \pm 0.39$ & $11.27 \pm 0.41$ & 0.000 & 0.000 & 0.000 \\
\hline L3-4 & $11.73 \pm 0.29$ & $11.57 \pm 0.17$ & $11.60 \pm 0.25$ & 0.000 & 0.000 & 0.047 \\
\hline$\lfloor 4-5$ & $11.66 \pm 0.37$ & $11.51 \pm 0.28$ & $11.55 \pm 0.39$ & 0.000 & 0.000 & 0.151 \\
\hline L5-S1 & $10.92 \pm 0.10$ & $10.77 \pm 0.10$ & $10.80 \pm 0.08$ & 0.000 & 0.000 & 0.016 \\
\hline
\end{tabular}

Values are presented as mean \pm standard deviation. A-B means initial versus 3 months; B-C means 3 months versus 6 months; A-C means initial versus 6 months. a) By repeated measures analysis of variance test. 
Table 11. Cross-sectional area \& fat content of lumbar muscles at disc level of lumbar spine initially and on follow-up ( $n=51)$

\begin{tabular}{|c|c|c|c|c|c|c|c|}
\hline \multirow{2}{*}{ Level } & \multirow{2}{*}{\multicolumn{2}{|c|}{ Lumbar muscles }} & \multicolumn{4}{|c|}{ Cross-sectional area $\left(\mathrm{cm}^{2}\right)$} & \multirow{2}{*}{ Intergroup comparisons } \\
\hline & & & Initial (A) & $3 \mathrm{mo}(\mathrm{B})$ & $6 \mathrm{mo}(\mathrm{C})$ & $p$-value ${ }^{\mathrm{a})}$ & \\
\hline \multirow[t]{12}{*}{ L1-L2 } & Multifidus & Left & $4.55 \pm 0.45$ & $4.07 \pm 0.27$ & $4.36 \pm 0.52$ & $<0.001$ & - \\
\hline & & Right & $4.33 \pm 0.42$ & $3.89 \pm 0.39$ & $4.05 \pm 0.55$ & $<0.001$ & $B-C=0.153$ \\
\hline & Erector spinae & Left & $9.78 \pm 0.62$ & $9.27 \pm 0.78$ & $9.39 \pm 0.49$ & 0.001 & $B-C=0.412$ \\
\hline & & Right & $9.69 \pm 0.53$ & $9.27 \pm 0.60$ & $9.65 \pm 0.77$ & 0.003 & $A-C=1.00$ \\
\hline & Ilio-psoas & Left & $6.65 \pm 0.39$ & $6.71 \pm 0.93$ & $6.57 \pm 0.36$ & 0.378 & $A-B=1.00, B-C=0.798, C-A=0.318$ \\
\hline & & Right & $6.54 \pm 0.37$ & $6.12 \pm 0.37$ & $6.19 \pm 0.48$ & $<0.001$ & $B-C=0.782$ \\
\hline & Quadratus lumborum & Left & $4.92 \pm 0.29$ & $4.70 \pm 0.45$ & $4.68 \pm 0.39$ & $<0.001$ & $B-C=1.00$ \\
\hline & & Right & $4.74 \pm 0.35$ & $4.45 \pm 0.46$ & $4.54 \pm 0.33$ & 0.002 & B-C- $=0.836$ \\
\hline & Rectus abdominis & Left & $4.94 \pm 0.38$ & $4.57 \pm 0.44$ & $4.69 \pm 0.47$ & $<0.001$ & - \\
\hline & & Right & $5.38 \pm 0.38$ & $5.07 \pm 0.26$ & $5.17 \pm 0.33$ & $<0.001$ & $B-C=0.192$ \\
\hline & Obliques & Left & $22.07 \pm 2.83$ & $19.55 \pm 2.96$ & $20.11 \pm 3.46$ & $<0.001$ & $B-C=0.893$ \\
\hline & & Right & $22.92 \pm 3.00$ & $18.87 \pm 2.92$ & $21.17 \pm 2.48$ & $<0.001$ & - \\
\hline \multirow[t]{12}{*}{ L2-L3 } & Multifidus & Left & $4.55 \pm 0.44$ & $4.10 \pm 0.29$ & $4.37 \pm 0.50$ & $<0.001$ & - \\
\hline & & Right & $4.31 \pm 0.43$ & $3.92 \pm 0.38$ & $4.05 \pm 0.55$ & $<0.001$ & $B-C=0.354$ \\
\hline & Erector spinae & Left & $10.09 \pm 1.02$ & $9.30 \pm 2.27$ & $9.51 \pm 0.73$ & 0.058 & $A-B=0.187, B-C=1.00$ \\
\hline & & Right & $10.29 \pm 1.19$ & $8.98 \pm 1.26$ & $10.01 \pm 0.95$ & $<0.001$ & $A-C=0.188$ \\
\hline & Ilio-psoas & Left & $6.66 \pm 0.39$ & $6.37 \pm 0.61$ & $6.57 \pm 0.36$ & 0.374 & $B-C=0.066, C-A=0.220$ \\
\hline & & Right & $6.55 \pm 0.37$ & $6.16 \pm 0.36$ & $6.21 \pm 0.48$ & $<0.00$ & $B-C=1.00$ \\
\hline & Quadratus lumborum & Left & $4.93 \pm 0.29$ & $4.68 \pm 0.40$ & $4.68 \pm 0.40$ & $<0.001$ & $B-C=1.00$ \\
\hline & & Right & $4.76 \pm 0.34$ & $4.47 \pm 0.40$ & $4.57 \pm 0.30$ & 0.002 & B-C $=0.398$ \\
\hline & Rectus abdominis & Left & $4.94 \pm 0.38$ & $4.53 \pm 0.45$ & $4.69 \pm 0.48$ & $<0.001$ & - \\
\hline & & Right & $5.38 \pm 0.38$ & $5.06 \pm 0.28$ & $5.16 \pm 0.33$ & $<0.001$ & $B-C=0.164$ \\
\hline & Obliques & Left & $22.14 \pm 2.99$ & $18.12 \pm 3.26$ & $20.54 \pm 3.17$ & $<0.001$ & - \\
\hline & & Right & $23.15 \pm 3.21$ & $18.73 \pm 3.57$ & $21.91 \pm 2.19$ & $<0.001$ & - \\
\hline \multirow[t]{12}{*}{ L3-4 } & Multifidus & Left & $4.56 \pm 0.43$ & $4.08 \pm 0.27$ & $4.33 \pm 0.52$ & $<0.001$ & - \\
\hline & & Right & $4.33 \pm 0.42$ & $3.89 \pm 0.39$ & $4.05 \pm 0.55$ & $<0.001$ & $B-C=0.153$ \\
\hline & Erector spinae & Left & $12.42 \pm 3.13$ & $12.27 \pm 3.60$ & $12.09 \pm 3.21$ & 0.325 & $A-B=1.00, B-C=1.00, A-C=0.073$ \\
\hline & & Right & $12.62 \pm 3.54$ & $11.75 \pm 3.03$ & $11.77 \pm 2.86$ & $<0.001$ & $B-C=1.00$ \\
\hline & Ilio-psoas & Left & $6.66 \pm 0.40$ & $6.39 \pm 0.69$ & $6.57 \pm 0.36$ & 0.374 & $B-C=0.155, A-C=0.206$ \\
\hline & & Right & $6.54 \pm 0.37$ & $6.11 \pm 0.37$ & $6.20 \pm 0.47$ & $<0.00$ & $B-C=0.332$ \\
\hline & Quadratus lumborum & Left & $4.92 \pm 0.29$ & $4.68 \pm 0.45$ & $4.68 \pm 0.39$ & $<0.001$ & $B-C=1.00$ \\
\hline & & Right & $4.74 \pm 0.35$ & $4.46 \pm 0.45$ & $4.55 \pm 0.33$ & 0.002 & - \\
\hline & Rectus abdominis & Left & $4.94 \pm 0.37$ & $4.56 \pm 0.45$ & $4.69 \pm 0.47$ & $<0.001$ & - \\
\hline & & Right & $5.40 \pm 0.35$ & $5.01 \pm 0.30$ & $5.16 \pm 0.32$ & $<0.001$ & - \\
\hline & Obliques & Left & $21.87 \pm 2.89$ & $18.58 \pm 3.14$ & $19.92 \pm 3.82$ & $<0.001$ & - \\
\hline & & Right & $22.86 \pm 3.14$ & $19.05 \pm 3.28$ & $22.07 \pm 2.25$ & $<0.001$ & - \\
\hline \multirow[t]{4}{*}{ L4-L5 } & Multifidus & Left & $7.44 \pm 1.40$ & $6.10 \pm 1.14$ & $7.35 \pm 1.42$ & $<0.001$ & $A-C=1.00$ \\
\hline & & Right & $7.12 \pm 1.41$ & $6.31 \pm 1.27$ & $7.00 \pm 1.42$ & $<0.001$ & $A-C=1.00$ \\
\hline & Erector spinae & Left & $9.79 \pm 0.62$ & $9.27 \pm 0.55$ & $9.38 \pm 0.50$ & 0.001 & $B-C=0.376$ \\
\hline & & Right & $9.72 \pm 0.53$ & $9.32 \pm 0.50$ & $9.50 \pm 0.52$ & 0.003 & $B-C=0.176$ \\
\hline
\end{tabular}


Table 11. Continued

\begin{tabular}{|c|c|c|c|c|c|c|c|}
\hline \multirow{2}{*}{ Level } & \multirow{2}{*}{\multicolumn{2}{|c|}{ Lumbar muscles }} & \multicolumn{4}{|c|}{ Cross-sectional area $\left(\mathrm{cm}^{2}\right)$} & \multirow{2}{*}{ Intergroup comparisons } \\
\hline & & & Initial (A) & $3 \mathrm{mo}(\mathrm{B})$ & $6 \mathrm{mo}(\mathrm{C})$ & $p$-value ${ }^{\text {a) }}$ & \\
\hline & \multirow[t]{2}{*}{ Ilio-psoas } & Left & $6.65 \pm 0.39$ & $6.47 \pm 0.61$ & $6.58 \pm 0.38$ & 0.373 & $B-C=0.354, A-C=0.541$ \\
\hline & & Right & $6.54 \pm 0.36$ & $6.11 \pm 0.37$ & $6.20 \pm 0.38$ & $<0.001$ & $B-C=0.227$ \\
\hline & \multirow[t]{2}{*}{ Quadratus lumborum } & Left & $4.94 \pm 0.30$ & $4.65 \pm 0.46$ & $4.67 \pm 0.40$ & $<0.001$ & $B-C=1.00$ \\
\hline & & Right & $4.76 \pm 0.33$ & $4.47 \pm 0.45$ & $4.53 \pm 0.33$ & 0.003 & $B-C=1.00$ \\
\hline & \multirow[t]{2}{*}{ Rectus abdominis } & Left & $4.95 \pm 0.37$ & $4.53 \pm 0.45$ & $4.68 \pm 0.47$ & $<0.001$ & - \\
\hline & & Right & $5.37 \pm 0.39$ & $5.05 \pm 0.29$ & $5.17 \pm 0.33$ & $<0.001$ & $B-C=0.110$ \\
\hline & \multirow[t]{2}{*}{ Obliques } & Left & $22.22 \pm 2.85$ & $19.36 \pm 2.66$ & $19.95 \pm 3.51$ & $<0.001$ & $B-C=0.434$ \\
\hline & & Right & $23.20 \pm 2.63$ & $19.53 \pm 3.99$ & $21.27 \pm 2.70$ & $<0.001$ & - \\
\hline \multirow[t]{8}{*}{ L5-S1 } & \multirow[t]{2}{*}{ Multifidus } & Left & $9.39 \pm 0.95$ & $9.01 \pm 0.86$ & $9.14 \pm 0.95$ & 0.325 & $B-C=0.171$ \\
\hline & & Right & $9.53 \pm 0.89$ & $8.90 \pm 1.27$ & $9.06 \pm 1.01$ & $<0.001$ & $B-C=0.876$ \\
\hline & \multirow[t]{2}{*}{ Erector spinae } & Left & $9.79 \pm 0.62$ & $9.40 \pm 0.58$ & $9.43 \pm 0.43$ & 0.001 & $B-C=1.00$ \\
\hline & & Right & $9.69 \pm 0.52$ & $9.26 \pm 0.60$ & $9.51 \pm 0.57$ & 0.003 & $B-C=0.085, C-A=0.171$ \\
\hline & \multirow[t]{2}{*}{ Ilio-psoas } & Left & $16.37 \pm 1.18$ & $14.77 \pm 1.13$ & $14.74 \pm 1.47$ & $<0.001$ & $B-C=1.00$ \\
\hline & & Right & $14.64 \pm 1.92$ & $13.38 \pm 2.15$ & $14.09 \pm 1.70$ & $<0.001$ & - \\
\hline & \multirow[t]{2}{*}{ Rectus abdominis } & Left & $4.94 \pm 0.38$ & $4.53 \pm 0.46$ & $4.69 \pm 0.47$ & $<0.001$ & - \\
\hline & & Right & $5.38 \pm 0.38$ & $5.00 \pm 0.31$ & $5.15 \pm 0.33$ & $<0.001$ & - \\
\hline
\end{tabular}

Values are presented as mean \pm standard deviation. A-B means initial versus 3 months; B-C means 3 months versus 6 months; A-C means initial versus 6 months. Intergroup comparisons are all significant except these below mentioned.

${ }^{a}$ By repeated measures analysis of variance test.

CSA of the lumbar muscles at the follow-up compared with that at admission. When the CSA of the lumbar muscles was analyzed according to the type of management (operative/conservative), no significant differences were found in the majority of muscles, except for the right erector spinae and left rectus abdominis at the L1-L2 level at the 3-month follow-up compared with at admission (Table 12). When the CSA of the lumbar muscles was analyzed according to the severity of the initial injury, no significant differences were noted over time for most of the lumbar muscles according to posthoc analysis (Tables 13-17).

Fig. 1 shows measurements of cross sectional area of trunk muscles on axial T2 weighted images; fig. 2 shows measurements of trunk depth and width on axial T2 weighted images at lumbar disc levels.

\section{Discussion}

The asymmetry of the size and composition of the paraspinal muscle has been reported in patients with a clinical presentation of unilateral low back pain (LBP) with or without radiculopathy $[6,7,9,10]$. Singh et al. [4] observed that in patients with chronic LBP, trunk width, depth, and skin angle were comparable at the L3-L4, L4-L5, and L5S1 disc levels. By contrast, significant differences were observed between patients with LBP and healthy volunteers regarding disc angle at the $\mathrm{L} 3-\mathrm{L} 4(p=0.005)$ and $\mathrm{L} 4-$ L5 levels $(p=0.02)$ and in the CSA of the disc at the L4-L5 level $(p=0.01)$. The CSA of the paraspinal and abdominal oblique muscles tended to be smaller among patients, but this was not statistically different to that of healthy volunteers. These studies indicate that paravertebral muscles respond to changes in the normal biomechanics or anatomy of the vertebral column [4,6-10]. The present study supports these studies by providing corroborative data from patients with SCI in whom biomechanical and anatomical changes occurred after the traumatic event. We also highlight the fact that even normal discs distal to the lesion respond to the traumatic event, thus resulting in decreased disc angle, disc CSA, and skin angle. The factors underlying these changes may include altered biomechanics, abnormal stresses, and changes in the nutrition of the discs.

We observed significant decreases in trunk depth and 
Table 12. CSA \& fat content of lumbar muscles at disc level of lumbar spine initially and on follow-up according to their type of management ( $\mathrm{n}=51$ )

\begin{tabular}{|c|c|c|c|c|c|c|c|c|c|c|c|}
\hline \multirow{3}{*}{$\begin{array}{l}\text { Level } \\
\text { L1-L2 }\end{array}$} & \multirow{2}{*}{\multicolumn{2}{|c|}{$\begin{array}{l}\text { Mean CSA }\left(\mathrm{cm}^{2}\right) \text { of } \\
\text { lumbar muscles }\end{array}$}} & \multicolumn{3}{|c|}{ Initial } & \multicolumn{3}{|c|}{$3 \mathrm{mo}$} & \multicolumn{3}{|c|}{$6 \mathrm{mo}$} \\
\hline & & & \multirow{2}{*}{$\begin{array}{c}\text { Operative } \\
\text { (n=35) }\end{array}$} & \multirow{2}{*}{$\begin{array}{l}\text { Conservative } \\
\qquad(\mathrm{n}=16) \\
4.54 \pm 0.47\end{array}$} & \multirow{2}{*}{$\begin{array}{c}p \text {-value }{ }^{\text {a) }} \\
0.972\end{array}$} & \multirow{2}{*}{$\begin{array}{c}\text { Operative } \\
\text { (n=35) } \\
4.08 \pm 0.27\end{array}$} & \multirow{2}{*}{$\begin{array}{l}\text { Conservative } \\
\text { (n=16) } \\
4.06 \pm 0.29\end{array}$} & \multirow{2}{*}{$\begin{array}{c}p \text {-value }{ }^{\text {a) }} \\
0.860\end{array}$} & \multirow{2}{*}{$\begin{array}{c}\text { Operative } \\
\text { (n=35) } \\
4.39 \pm 0.48\end{array}$} & \multirow{2}{*}{$\begin{array}{l}\text { Conservative } \\
\qquad(\mathrm{n}=16) \\
4.31 \pm 0.60\end{array}$} & \multirow{2}{*}{$\begin{array}{c}p \text {-value }{ }^{\text {a) }} \\
0.605\end{array}$} \\
\hline & Multifidus & Left & & & & & & & & & \\
\hline & & Right & $4.38 \pm 0.48$ & $4.21 \pm 0.22$ & 0.100 & $3.96 \pm 0.41$ & $3.74 \pm 0.31$ & 0.055 & $4.15 \pm 0.59$ & $3.84 \pm 0.37$ & 0.066 \\
\hline & Erector spinae & Left & $9.72 \pm 0.52$ & $9.90 \pm 0.80$ & 0.349 & $9.26 \pm 0.63$ & $9.27 \pm 1.06$ & 0.980 & $9.44 \pm 0.40$ & $9.28 \pm 0.64$ & 0.285 \\
\hline & & Right & $9.65 \pm 0.55$ & $9.78 \pm 0.49$ & 0.433 & $9.15 \pm 0.67$ & $9.51 \pm 0.33$ & 0.013 & $9.51 \pm 0.51$ & $9.97 \pm 1.12$ & 0.133 \\
\hline & Ilio-psoas & Left & $6.62 \pm 0.41$ & $6.71 \pm 0.33$ & 0.482 & $6.73 \pm 1.09$ & $6.66 \pm 0.43$ & 0.717 & $6.55 \pm 0.30$ & $6.61 \pm 0.48$ & 0.562 \\
\hline & & Right & $6.55 \pm 0.39$ & $6.53 \pm 0.33$ & 0.855 & $6.14 \pm 0.37$ & $6.09 \pm 0.40$ & 0.646 & $6.21 \pm 0.52$ & $6.16 \pm 0.41$ & 0.738 \\
\hline & Quadratus lumborum & Left & $4.89 \pm 0.27$ & $5.00 \pm 0.32$ & 0.204 & $4.66 \pm 0.44$ & $4.79 \pm 0.49$ & 0.333 & $4.65 \pm 0.41$ & $4.76 \pm 0.37$ & 0.360 \\
\hline & & Right & $4.74 \pm 0.36$ & $4.73 \pm 0.33$ & 0.888 & $4.46 \pm 0.50$ & $4.44 \pm 0.36$ & 0.924 & $4.56 \pm 0.36$ & $4.51 \pm 0.27$ & 0.615 \\
\hline & Rectus abdominis & Left & $5.00 \pm 0.37$ & $4.79 \pm 0.34$ & 0.056 & $4.66 \pm 0.43$ & $4.36 \pm 0.40$ & 0.021 & $4.75 \pm 0.44$ & $4.54 \pm 0.50$ & 0.125 \\
\hline & & Right & $5.34 \pm 0.55$ & $5.47 \pm 0.42$ & 0.255 & $5.05 \pm 0.24$ & $5.11 \pm 0.32$ & 0.451 & $5.13 \pm 0.30$ & $5.24 \pm 0.38$ & 0.251 \\
\hline & Obliques & Left & $22.53 \pm 2.78$ & $21.06 \pm 2.75$ & 0.085 & $19.61 \pm 2.74$ & $19.40 \pm 3.48$ & 0.815 & $20.74 \pm 3.42$ & $18.73 \pm 0.48$ & 0.053 \\
\hline & & Right & $22.78 \pm 3.10$ & $18.66 \pm 2.99$ & 0.638 & $18.66 \pm 2.99$ & $19.31 \pm 2.80$ & 0.466 & $21.14 \pm 2.56$ & $21.23 \pm 2.39$ & 0.911 \\
\hline \multirow[t]{12}{*}{ L2-3 } & Multifidus & Left & $4.55 \pm 0.43$ & $4.56 \pm 0.45$ & 0.972 & $4.09 \pm 0.28$ & $4.11 \pm 0.31$ & 0.812 & $4.40 \pm 0.48$ & $4.32 \pm 0.53$ & 0.605 \\
\hline & & Right & $4.35 \pm 0.49$ & $4.23 \pm 0.20$ & 0.100 & $3.98 \pm 0.43$ & $3.80 \pm 0.22$ & 0.125 & $4.15 \pm 0.59$ & $3.84 \pm 0.37$ & 0.066 \\
\hline & Erector spinae & Left & $10.14 \pm 1.04$ & $9.99 \pm 1.00$ & 0.349 & $9.33 \pm 2.37$ & $9.26 \pm 2.09$ & 0.920 & $9.57 \pm 0.72$ & $9.38 \pm 0.76$ & 0.387 \\
\hline & & Right & $10.27 \pm 1.01$ & $10.34 \pm 1.56$ & 0.433 & $8.98 \pm 1.08$ & $8.96 \pm 1.62$ & 0.945 & $9.87 \pm 0.74$ & $10.33 \pm 1.28$ & 0.190 \\
\hline & Ilio-psoas & Left & $6.63 \pm 0.41$ & $6.73 \pm 0.34$ & 0.482 & $6.28 \pm 0.69$ & $6.56 \pm 0.34$ & 0.121 & $6.56 \pm 0.31$ & $6.58 \pm 0.48$ & 0.869 \\
\hline & & Right & $6.55 \pm 0.39$ & $6.54 \pm 0.33$ & 0.855 & $6.13 \pm 0.37$ & $6.23 \pm 0.35$ & 0.366 & $6.20 \pm 0.51$ & $6.22 \pm 0.39$ & 0.898 \\
\hline & Quadratus lumborum & Left & $4.90 \pm 0.28$ & $5.00 \pm 0.32$ & 0.204 & $4.63 \pm 0.35$ & $4.78 \pm 0.48$ & 0.199 & $4.65 \pm 0.41$ & $4.75 \pm 0.38$ & 0.403 \\
\hline & & Right & $4.77 \pm 0.35$ & $4.73 \pm 0.33$ & 0.888 & $4.48 \pm 0.41$ & $4.44 \pm 0.36$ & 0.783 & $4.60 \pm 0.31$ & $4.51 \pm 0.27$ & 0.322 \\
\hline & Rectus abdominis & Left & $5.00 \pm 0.38$ & $4.81 \pm 0.36$ & 0.056 & $4.64 \pm 0.43$ & $4.28 \pm 0.38$ & 0.006 & $4.76 \pm 0.45$ & $4.52 \pm 0.52$ & 0.090 \\
\hline & & Right & $5.34 \pm 0.36$ & $5.47 \pm 0.42$ & 0.255 & $5.04 \pm 0.26$ & $5.11 \pm 0.32$ & 0.412 & $5.13 \pm 0.30$ & $5.23 \pm 0.38$ & 0.319 \\
\hline & Obliques & Left & $22.72 \pm 2.90$ & $20.88 \pm 2.89$ & 0.085 & $18.44 \pm 3.10$ & $17.42 \pm 3.60$ & 0.306 & $21.03 \pm 2.98$ & $19.48 \pm 3.42$ & 0.105 \\
\hline & & Right & $22.79 \pm 3.17$ & $23.95 \pm 3.25$ & 0.638 & $17.70 \pm 3.04$ & $20.99 \pm 3.67$ & 0.002 & $21.60 \pm 2.18$ & $22.60 \pm 2.11$ & 0.131 \\
\hline \multirow[t]{12}{*}{ L3-L4 } & Multifidus & Left & $4.55 \pm 0.44$ & $4.57 \pm 0.44$ & 0.896 & $4.07 \pm 0.26$ & $4.10 \pm 0.31$ & 0.759 & $4.34 \pm 0.47$ & $4.29 \pm 0.62$ & 0.727 \\
\hline & & Right & $4.38 \pm 0.48$ & $4.21 \pm 0.23$ & 0.100 & $3.96 \pm 0.41$ & $3.74 \pm 0.31$ & 0.055 & $4.15 \pm 0.59$ & $3.84 \pm 0.37$ & 0.066 \\
\hline & Erector spinae & Left & $12.83 \pm 3.39$ & $11.51 \pm 2.32$ & 0.112 & $12.55 \pm 3.86$ & $11.65 \pm 2.98$ & 0.369 & $12.46 \pm 3.50$ & $11.28 \pm 2.34$ & 0.162 \\
\hline & & Right & $13.15 \pm 3.84$ & $11.46 \pm 2.51$ & 0.069 & $12.25 \pm 3.35$ & $10.66 \pm 1.83$ & 0.034 & $12.23 \pm 3.16$ & $10.75 \pm 1.78$ & 0.038 \\
\hline & Ilio-psoas & Left & $6.64 \pm 0.43$ & $6.71 \pm 0.33$ & 0.568 & $6.30 \pm 0.77$ & $6.59 \pm 0.42$ & 0.157 & $6.55 \pm 0.31$ & $6.60 \pm 0.50$ & 0.693 \\
\hline & & Right & $6.54 \pm 0.39$ & $6.53 \pm 0.33$ & 0.918 & $6.13 \pm 0.36$ & $6.06 \pm 0.40$ & 0.527 & $6.21 \pm 0.51$ & $6.19 \pm 0.36$ & 0.917 \\
\hline & Quadratus lumborum & Left & $4.89 \pm 0.27$ & $5.00 \pm 0.32$ & 0.190 & $4.63 \pm 0.44$ & $4.78 \pm 0.47$ & 0.307 & $4.64 \pm 0.40$ & $4.74 \pm 0.37$ & 0.400 \\
\hline & & Right & $4.75 \pm 0.36$ & $4.73 \pm 0.33$ & 0.846 & $4.32 \pm 0.46$ & $4.33 \pm 0.33$ & 0.969 & $4.56 \pm 0.36$ & $4.52 \pm 0.25$ & 0.657 \\
\hline & Rectus abdominis & Left & $5.01 \pm 0.38$ & $4.79 \pm 0.34$ & 0.051 & $4.66 \pm 0.43$ & $4.34 \pm 0.41$ & 0.016 & $4.76 \pm 0.45$ & $4.53 \pm 0.50$ & 0.109 \\
\hline & & Right & $5.34 \pm 0.35$ & $5.52 \pm 0.32$ & 0.095 & $4.99 \pm 0.31$ & $5.07 \pm 0.29$ & 0.365 & $5.12 \pm 0.29$ & $5.24 \pm 0.38$ & 0.198 \\
\hline & Obliques & Left & $22.28 \pm 2.81$ & $20.96 \pm 2.96$ & 0.133 & $18.64 \pm 2.81$ & $18.46 \pm 3.85$ & 0.853 & $20.58 \pm 3.40$ & $18.49 \pm 4.40$ & 0.070 \\
\hline & & Right & $22.35 \pm 2.97$ & $23.97 \pm 3.32$ & 0.087 & $18.35 \pm 2.87$ & $20.58 \pm 3.71$ & 0.023 & $21.56 \pm 2.33$ & $23.18 \pm 1.64$ & 0.015 \\
\hline \multirow[t]{6}{*}{ L4-L5 } & Multifidus & Left & $7.43 \pm 1.51$ & $7.46 \pm 1.14$ & 0.959 & $6.23 \pm 1.27$ & $5.83 \pm 0.76$ & 0.169 & $7.45 \pm 1.50$ & $7.11 \pm 1.23$ & 0.430 \\
\hline & & Right & $7.25 \pm 1.56$ & $6.85 \pm 1.00$ & 0.353 & $6.48 \pm 1.42$ & $5.93 \pm 0.78$ & 0.083 & $7.11 \pm 1.54$ & $6.77 \pm 1.13$ & 0.434 \\
\hline & Erector spinae & Left & $9.73 \pm 0.52$ & $9.93 \pm 0.81$ & 0.314 & $9.20 \pm 0.56$ & $9.43 \pm 0.53$ & 0.180 & $9.43 \pm 0.43$ & $9.28 \pm 0.64$ & 0.338 \\
\hline & & Right & $9.66 \pm 0.55$ & $9.86 \pm 0.46$ & 0.221 & $9.23 \pm 0.55$ & $9.51 \pm 0.33$ & 0.029 & $9.51 \pm 0.51$ & $9.48 \pm 0.53$ & 0.877 \\
\hline & Ilio-psoas & Left & $6.62 \pm 0.42$ & $6.71 \pm 0.33$ & 0.453 & $6.41 \pm 0.68$ & $6.59 \pm 0.42$ & 0.338 & $6.56 \pm 0.33$ & $6.63 \pm 0.48$ & 0.559 \\
\hline & & Right & $6.54 \pm 0.39$ & $6.53 \pm 0.32$ & 0.917 & $6.14 \pm 0.36$ & $6.05 \pm 0.39$ & 0.425 & $6.21 \pm 0.38$ & $6.18 \pm 0.40$ & 0.755 \\
\hline
\end{tabular}


Table 12. Continued

\begin{tabular}{|c|c|c|c|c|c|c|c|c|c|c|c|}
\hline \multirow[b]{2}{*}{ Level } & \multirow{2}{*}{\multicolumn{2}{|c|}{$\begin{array}{c}\text { Mean CSA }\left(\mathrm{cm}^{2}\right) \text { of } \\
\text { lumbar muscles }\end{array}$}} & \multicolumn{3}{|c|}{ Initial } & \multicolumn{3}{|c|}{$3 \mathrm{mo}$} & \multicolumn{3}{|c|}{$6 \mathrm{mo}$} \\
\hline & & & $\begin{array}{l}\text { Operative } \\
(n=35)\end{array}$ & $\begin{array}{l}\text { Conservative } \\
\qquad(n=16)\end{array}$ & $p$-value ${ }^{\text {a) }}$ & $\begin{array}{l}\text { Operative } \\
\qquad(n=35)\end{array}$ & $\begin{array}{l}\text { Conservative } \\
\qquad(\mathrm{n}=16)\end{array}$ & $p$-value ${ }^{\text {a) }}$ & $\begin{array}{l}\text { Operative } \\
(\mathrm{n}=35)\end{array}$ & $\begin{array}{l}\text { Conservative } \\
\qquad(\mathrm{n}=16)\end{array}$ & $p$-value ${ }^{\text {a) }}$ \\
\hline & Quadratus lumborum & Left & $4.91 \pm 0.29$ & $5.00 \pm 0.33$ & 0.330 & $4.63 \pm 0.43$ & $4.69 \pm 0.51$ & 0.642 & $4.63 \pm 0.41$ & $4.75 \pm 0.39$ & 0.346 \\
\hline & & Right & $4.76 \pm 0.36$ & $4.77 \pm 0.27$ & 0.860 & $4.45 \pm 0.50$ & $4.49 \pm 0.35$ & 0.773 & $4.55 \pm 0.35$ & $4.49 \pm 0.27$ & 0.554 \\
\hline & Rectus abdominis & Left & $5.03 \pm 0.36$ & $4.79 \pm 0.34$ & 0.032 & $4.62 \pm 0.45$ & $4.36 \pm 0.40$ & 0.058 & $4.75 \pm 0.44$ & $4.54 \pm 0.50$ & 0.139 \\
\hline & & Right & $5.33 \pm 0.37$ & $5.47 \pm 0.42$ & 0.231 & $5.03 \pm 0.28$ & $5.08 \pm 0.33$ & 0.632 & $5.14 \pm 0.31$ & $5.23 \pm 0.37$ & 0.430 \\
\hline & Obliques & Left & $22.64 \pm 2.79$ & $21.29 \pm 2.86$ & 0.116 & $19.69 \pm 2.47$ & $18.63 \pm 2.99$ & 0.189 & $20.56 \pm 3.47$ & $18.63 \pm 3.33$ & 0.067 \\
\hline & & Right & $22.71 \pm 2.40$ & $24.28 \pm 2.87$ & 0.047 & $19.19 \pm 3.55$ & $20.28 \pm 4.86$ & 0.370 & $21.15 \pm 2.45$ & $21.52 \pm 3.26$ & 0.660 \\
\hline $\mathrm{L} 5-\mathrm{S} 1$ & Multifidus & Left & $9.53 \pm 0.95$ & $9.09 \pm 0.90$ & 0.124 & $9.11 \pm 0.91$ & $8.78 \pm 0.72$ & 0.212 & $9.18 \pm 0.97$ & $9.04 \pm 0.94$ & 0.617 \\
\hline & & Right & $9.65 \pm 0.95$ & $9.24 \pm 0.72$ & 0.129 & $8.83 \pm 1.23$ & $9.05 \pm 1.37$ & 0.562 & $9.15 \pm 1.00$ & $8.85 \pm 1.05$ & 0.330 \\
\hline & Erector spinae & Left & $9.73 \pm 0.52$ & $9.93 \pm 0.80$ & 0.284 & $9.32 \pm 0.54$ & $9.56 \pm 0.64$ & 0.172 & $9.43 \pm 0.43$ & $9.43 \pm 0.44$ & 0.984 \\
\hline & & Right & $9.65 \pm 0.54$ & $9.78 \pm 0.49$ & 0.451 & $9.15 \pm 0.66$ & $9.50 \pm 0.33$ & 0.014 & $9.51 \pm 0.52$ & $9.51 \pm 0.70$ & 0.977 \\
\hline & Ilio-psoas & Left & $16.36 \pm 1.30$ & $16.39 \pm 0.87$ & 0.950 & $14.66 \pm 1.18$ & $15.04 \pm 0.96$ & 0.300 & $14.62 \pm 1.55$ & $15.04 \pm 1.25$ & 0.365 \\
\hline & & Right & $14.47 \pm 1.98$ & $15.03 \pm 1.78$ & 0.369 & $13.14 \pm 2.23$ & $13.98 \pm 1.90$ & 0.223 & $13.88 \pm 1.80$ & $14.59 \pm 1.39$ & 0.147 \\
\hline & Rectus abdominis & Left & $5.01 \pm 0.37$ & $4.79 \pm 0.34$ & 0.047 & $4.64 \pm 0.44$ & $4.29 \pm 0.41$ & 0.008 & $4.76 \pm 0.45$ & $4.54 \pm 0.50$ & 0.123 \\
\hline & & Right & $5.34 \pm 0.35$ & $5.47 \pm 0.42$ & 0.273 & $4.99 \pm 0.31$ & $5.03 \pm 0.32$ & 0.635 & $5.11 \pm 0.30$ & $5.23 \pm 0.38$ & 0.237 \\
\hline
\end{tabular}

Values are presented as mean \pm standard deviation.

CSA, cross-sectional area

${ }^{a}$ By independent Student $t$-test.
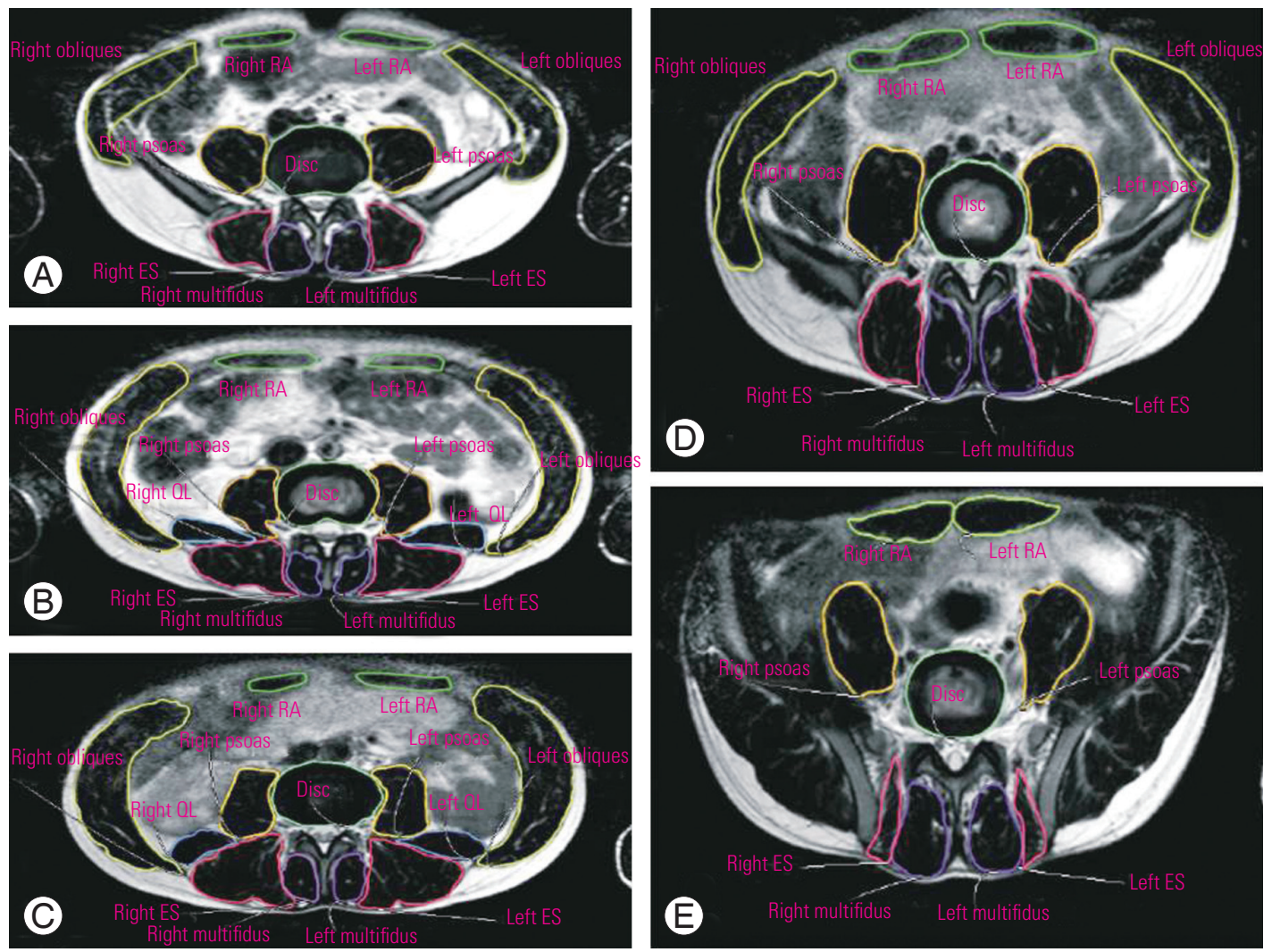

Fig. 1. Measurements of the cross-sectional areas of trunk muscles on axial T2-weighted images at the (A) $L 1-L 2$, (B) $L 2-L 3$, (C) $L 3-L 4$, (D) L4-L5, and (E) L5-S1 lumbar disc levels. RA, rectus abdominis; ES, erector spinae; $Q L$, quadratus lumborum. 

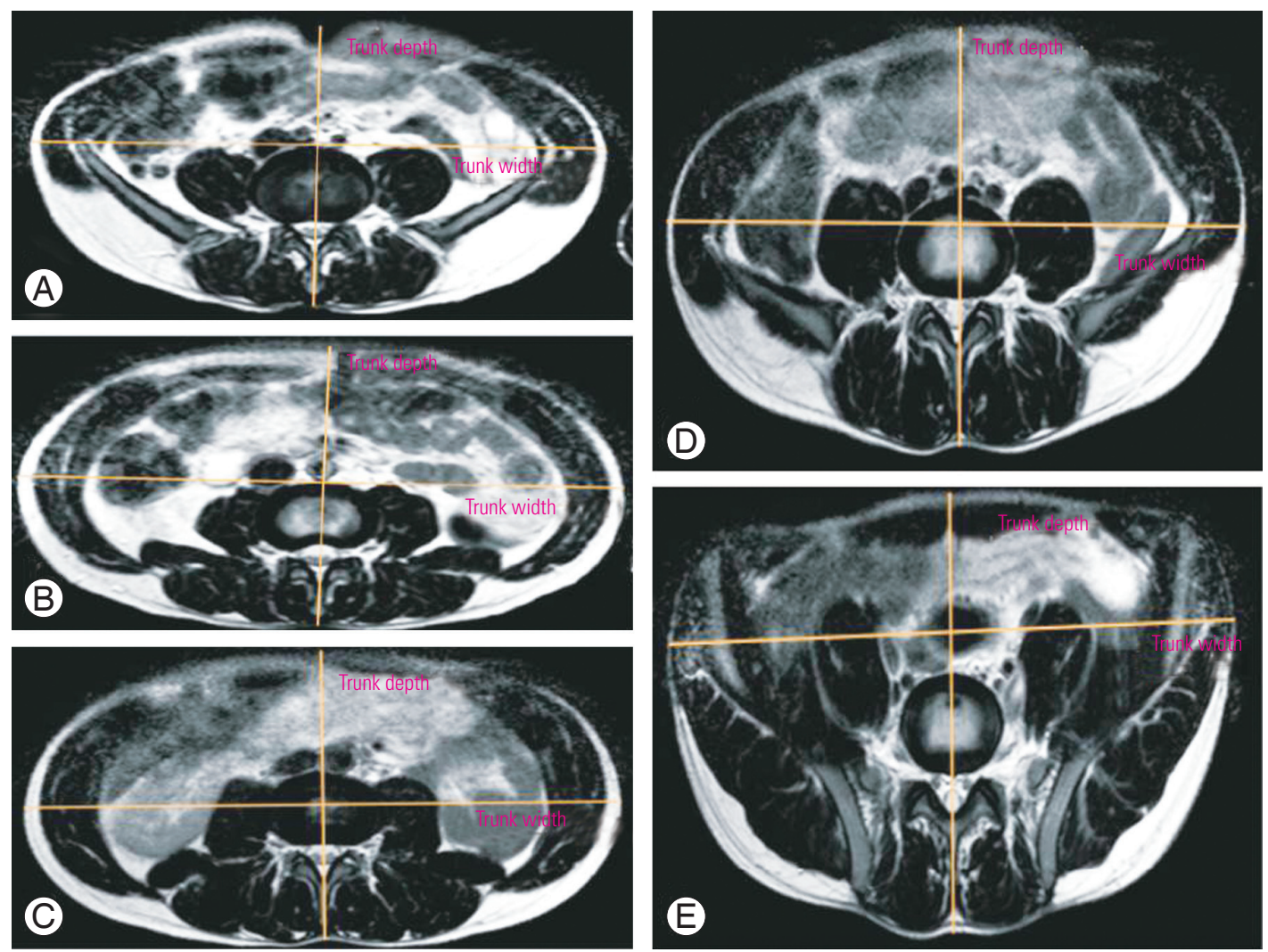

Fig. 2. Measurements of trunk depth and width on axial T2-weighted images at the (A) L1-L2, (B) L2-L3, (C) L3-L4, (D) L4-L5, and (E) L5S1 lumbar disc levels.

width at most levels of the spinal cord at the 3-month follow-up after SCI compared with that at admission, thus demonstrating the response of the paraspinal muscles to SCI. The decrease in mean CSA of all the lumbar muscles and subsequent increase might be due to the neurological improvement and active rehabilitation of the patient. As weight bearing begins and neurological improvement occurs, the observed changes may begin to revert to normal levels in response to the restoration of the normal biomechanics and anatomical alignment of the vertebral column. Many previous studies have highlighted the need for early rehabilitation and weight bearing (if possible) after the initial management of vertebral column injuries $[11,12]$. The limited strength observed in patients with SCI may be due to neurological factors or the insufficient muscle mass (more accurately, insufficient physiological muscle CSA) of neurally intact muscles. The neurologically intact muscles of a person with SCI are likely to respond to strength in the same way as those of an able-bodied person [5]. Indeed, clinical trials involving patients with SCI have demonstrated that progressive resistance training for nonparalyzed muscles increases the strength and quality of life of the patients $[13,14]$. It is not clear why partially paralyzed muscles are directly affected by SCI, although there is strong evidence to indicate that people who suffer partial paralysis following SCI become stronger over time [15].

Clinical trials and nonrandomized studies have consistently demonstrated that the strength of partially paralyzed muscles increases over time. It is generally assumed that this is due to a combination of central and peripheral factors. Peripheral factors include muscle hypertrophy, whereas central factors include adaptations either at the site of SCI or within the brain. It is unclear how much of the observed increase in the strength of partially paralyzed muscles can be attributed to physiotherapy intervention as opposed to natural recovery [5]. It has been reported that reversing the changes in muscles or at least restoring the normal proportions of fat and contractile tissue in the cervical spine muscles may contribute to the improvement of the functional rate of recovery in chronic whiplash injury [5]. The reinnervation of denervated paraspinal muscles has been reported following posterior spinal surgery in patients with lumbar degenerative 
Table 13. CSA \& fat content of lumbar muscles at L1-L2 disc level of lumbar spine according to neurological status in terms of complete and incomplete injury (n=51)

\begin{tabular}{|c|c|c|c|c|c|c|c|}
\hline \multirow[b]{2}{*}{ Duration } & \multirow{2}{*}{\multicolumn{2}{|c|}{$\begin{array}{l}\text { CSA }\left(\mathrm{cm}^{2}\right) \text { of lumbar } \\
\text { muscles (initially) }\end{array}$}} & \multicolumn{4}{|c|}{ Severity of initial injury } & \multirow[b]{2}{*}{ Intergroup comparisons } \\
\hline & & & $\begin{array}{l}\text { Complete SCI } \\
\text { (ASIA grade A) } \\
(n=10)\end{array}$ & $\begin{array}{c}\text { Incomplete SCI } \\
\text { (ASIA grade B, C, D) } \\
(\mathrm{n}=39)\end{array}$ & $\begin{array}{c}\text { No deficit } \\
\text { (ASIA grade E) } \\
(n=2)\end{array}$ & $p$-vale ${ }^{\text {a) }}$ & \\
\hline \multirow[t]{12}{*}{ Initial } & Multifidus & Left & $4.67 \pm 0.42$ & $4.52 \pm 0.46$ & $4.40 \pm 0.28$ & 0.655 & Insignificant \\
\hline & & Right & $4.38 \pm 0.41$ & $4.30 \pm 0.43$ & $4.65 \pm 0.35$ & 0.411 & Insignificant \\
\hline & Erector spinae & Left & $10.36 \pm 0.86$ & $9.64 \pm 0.47$ & $9.60 \pm 0.00$ & 0.245 & Insignificant \\
\hline & & Right & $9.82 \pm 0.71$ & $9.65 \pm 0.48$ & $9.85 \pm 0.35$ & 0.500 & Insignificant \\
\hline & Ilio-psoas & Left & $6.63 \pm 0.44$ & $6.65 \pm 0.39$ & $6.70 \pm 0.00$ & 0.279 & Insignificant \\
\hline & & Right & $6.34 \pm 0.50$ & $6.539 \pm 0.32$ & $6.55 \pm 0.50$ & 0.679 & Insignificant \\
\hline & Quadratus lumborum & Left & $4.81 \pm 0.23$ & $4.97 \pm 0.30$ & $4.65 \pm 0.07$ & 0.205 & Insignificant \\
\hline & & Right & $4.46 \pm 0.38$ & $4.80 \pm 0.31$ & $4.90 \pm 0.28$ & 0.261 & Insignificant \\
\hline & Rectus abdominis & Left & $4.95 \pm 0.43$ & $4.92 \pm 0.37$ & $5.10 \pm 0.00$ & 0.117 & Insignificant \\
\hline & & Right & $5.35 \pm 0.18$ & $5.36 \pm 0.41$ & $5.90 \pm 0.00$ & 0.145 & Insignificant \\
\hline & Obliques & Left & $21.44 \pm 3.27$ & $22.21 \pm 2.79$ & $22.50 \pm 1.70$ & 0.377 & Insignificant \\
\hline & & Right & $23.17 \pm 3.08$ & $22.57 \pm 2.81$ & $22.92 \pm 3.00$ & 0.851 & Insignificant \\
\hline \multirow[t]{12}{*}{$3 \mathrm{mo}$} & Multifidus & Left & $3.99 \pm 0.18$ & $4.10 \pm 0.29$ & $3.95 \pm 0.35$ & 0.256 & Insignificant \\
\hline & & Right & $3.87 \pm 0.23$ & $3.90 \pm 0.43$ & $3.95 \pm 0.21$ & 0.816 & Insignificant \\
\hline & Erector spinae & Left & $8.98 \pm 1.15$ & $9.33 \pm 0.68$ & $9.50 \pm 0.00$ & 0.519 & Insignificant \\
\hline & & Right & $9.34 \pm 0.55$ & $9.24 \pm 0.64$ & $9.45 \pm 0.21$ & 0.036 & $A-B=0.031$ \\
\hline & Ilio-psoas & Left & $6.49 \pm 1.12$ & $6.75 \pm 0.90$ & $6.95 \pm 0.78$ & 0.903 & Insignificant \\
\hline & & Right & $5.98 \pm 0.55$ & $6.16 \pm 0.32$ & $6.20 \pm 0.28$ & 0.993 & Insignificant \\
\hline & Quadratus lumborum & Left & $4.66 \pm 0.34$ & $4.74 \pm 0.46$ & $4.10 \pm 0.57$ & 0.347 & Insignificant \\
\hline & & Right & $4.81 \pm 0.43$ & $4.36 \pm 0.43$ & $4.40 \pm 0.42$ & 0.042 & $A-B=0.039$ \\
\hline & Rectus abdominis & Left & $4.72 \pm 0.55$ & $4.53 \pm 0.42$ & $4.50 \pm 0.00$ & 0.040 & $B-C=0.035$ \\
\hline & & Right & $5.03 \pm 0.16$ & $5.09 \pm 0.29$ & $4.85 \pm 0.07$ & 0.033 & $A-C=0.034$ \\
\hline & Obliques & Left & $19.18 \pm 2.61$ & $19.49 \pm 3.06$ & $22.50 \pm 0.14$ & 0.796 & Insignificant \\
\hline & & Right & $19.80 \pm 2.37$ & $18.63 \pm 2.71$ & $18.90 \pm 9.19$ & 0.320 & Insignificant \\
\hline \multirow[t]{12}{*}{$6 \mathrm{mo}$} & Multifidus & Left & $4.22 \pm 0.21$ & $4.41 \pm 0.58$ & $4.15 \pm 0.07$ & 0.559 & Insignificant \\
\hline & & Right & $4.02 \pm 0.77$ & $4.06 \pm 0.51$ & $4.10 \pm 0.14$ & 0.259 & Insignificant \\
\hline & Erector spinae & Left & $9.16 \pm 0.65$ & $9.44 \pm 0.44$ & $9.60 \pm 0.00$ & 0.898 & Insignificant \\
\hline & & Right & $9.44 \pm 0.56$ & $9.73 \pm 0.82$ & $9.25 \pm 0.78$ & 0.346 & Insignificant \\
\hline & Ilio-psoas & Left & $6.45 \pm 0.30$ & $6.59 \pm 0.38$ & $6.70 \pm 0.14$ & 0.002 & $B-C=0.002, A-C=0.002$ \\
\hline & & Right & $5.85 \pm 0.54$ & $6.27 \pm 0.44$ & $6.25 \pm 0.50$ & 0.880 & Insignificant \\
\hline & Quadratus lumborum & Left & $4.33 \pm 0.56$ & $4.78 \pm 0.29$ & $4.45 \pm 0.07$ & 0.233 & Insignificant \\
\hline & & Right & $4.41 \pm 0.38$ & $4.57 \pm 0.32$ & $4.60 \pm 0.28$ & 0.553 & Insignificant \\
\hline & Rectus abdominis & Left & $4.81 \pm 0.40$ & $4.65 \pm 0.48$ & $4.85 \pm 0.78$ & 0.020 & $B-C=0.017, A-C=0.031$ \\
\hline & & Right & $5.13 \pm 0.16$ & $5.16 \pm 0.36$ & $5.50 \pm 0.00$ & 0.227 & Insignificant \\
\hline & Obliques & Left & $20.66 \pm 2.52$ & $19.90 \pm 3.73$ & $21.50 \pm 2.26$ & 0.481 & Insignificant \\
\hline & & Right & $21.00 \pm 2.56$ & $21.03 \pm 2.43$ & $24.65 \pm 0.50$ & 0.288 & Insignificant \\
\hline
\end{tabular}

Values are presented as mean \pm standard deviation. A-B means complete SCl versus incomplete SCl; B-C means incomplete SCI versus no deficit; A-C means complete $\mathrm{SCl}$ versus no deficit. Intergroup comparisons are all significant except below mentioned.

CSA, cross-sectional area; SCI, spinal cord injury; ASIA, American Spinal Injury Association.

${ }^{a}$ By one way analysis of variance test. 
Table 14. CSA \& fat content of lumbar muscles at L2-L3 disc level of lumbar spine according to initial neurological status in terms of complete and incomplete injury $(n=51)$

\begin{tabular}{|c|c|c|c|c|c|c|c|}
\hline \multirow[b]{2}{*}{ Duration } & \multirow{2}{*}{\multicolumn{2}{|c|}{$\begin{array}{l}\text { CSA }\left(\mathrm{cm}^{2}\right) \text { of lumbar } \\
\text { muscles (initially) }\end{array}$}} & \multicolumn{4}{|c|}{ Severity of initial injury } & \multirow[b]{2}{*}{ Intergroup comparison } \\
\hline & & & $\begin{array}{c}\text { Complete SCI } \\
\text { (ASIA grade A) } \\
(n=10)\end{array}$ & $\begin{array}{c}\text { Incomplete SCI } \\
\text { (ASIA grade B, C, D) } \\
(n=39)\end{array}$ & $\begin{array}{c}\text { No deficit } \\
\text { (ASIA grade E) } \\
(n=2)\end{array}$ & $p$-value ${ }^{\text {a) }}$ & \\
\hline \multirow[t]{12}{*}{ Initial } & Multifidus & Left & $4.67 \pm 0.42$ & $4.53 \pm 0.45$ & $4.40 \pm 0.28$ & 0.655 & Insignificant \\
\hline & & Right & $4.37 \pm 0.40$ & $4.28 \pm 0.43$ & $4.65 \pm 0.35$ & 0.729 & Insignificant \\
\hline & Erector spinae & Left & $10.36 \pm 0.86$ & $10.11 \pm 0.99$ & $10.45 \pm 1.63$ & 0.796 & Insignificant \\
\hline & & Right & $9.82 \pm 0.71$ & $10.42 \pm 1.29$ & $10.35 \pm 0.07$ & 0.278 & Insignificant \\
\hline & Ilio-psoas & Left & $6.63 \pm 0.44$ & $6.66 \pm 0.39$ & $6.70 \pm 0.00$ & 0.266 & Insignificant \\
\hline & & Right & $6.34 \pm 0.50$ & $6.60 \pm 0.32$ & $6.55 \pm 0.50$ & 0.689 & Insignificant \\
\hline & Quadratus lumborum & Left & $4.81 \pm 0.23$ & $4.97 \pm 0.30$ & $4.65 \pm 0.07$ & 0.238 & Insignificant \\
\hline & & Right & $4.57 \pm 0.40$ & $4.80 \pm 0.31$ & $4.90 \pm 0.28$ & 0.272 & Insignificant \\
\hline & Rectus abdominis & Left & $4.95 \pm 0.43$ & $4.93 \pm 0.38$ & $5.10 \pm 0.00$ & 0.115 & Insignificant \\
\hline & & Right & $5.35 \pm 0.18$ & $5.36 \pm 0.41$ & $5.90 \pm 0.00$ & 0.145 & Insignificant \\
\hline & Obliques & Left & $21.83 \pm 3.75$ & $22.20 \pm 2.89$ & $22.50 \pm 1.70$ & 0.472 & Insignificant \\
\hline & & Right & $23.17 \pm 3.08$ & $22.88 \pm 3.13$ & $28.35 \pm 0.07$ & 0.987 & Insignificant \\
\hline \multirow[t]{12}{*}{$3 \mathrm{mo}$} & Multifidus & Left & $4.08 \pm 0.25$ & $4.11 \pm 0.30$ & $3.95 \pm 0.35$ & 0.370 & Insignificant \\
\hline & & Right & $3.87 \pm 0.23$ & $3.93 \pm 0.42$ & $3.95 \pm 0.21$ & 0.483 & Insignificant \\
\hline & Erector spinae & Left & $8.98 \pm 1.15$ & $9.13 \pm 1.98$ & $14.25 \pm 6.72$ & 0.105 & Insignificant \\
\hline & & Right & $9.34 \pm 0.55$ & $8.85 \pm 1.39$ & $14.60 \pm 0.00$ & 0.001 & $A-B=0.034, B-C=0.003, A-C=0.038$ \\
\hline & Ilio-psoas & Left & $6.49 \pm 1.12$ & $6.34 \pm 0.43$ & $6.35 \pm 0.07$ & 0.455 & Insignificant \\
\hline & & Right & $5.97 \pm 0.54$ & $6.20 \pm 0.30$ & $6.20 \pm 0.28$ & 0.976 & Insignificant \\
\hline & Quadratus lumborum & Left & $4.63 \pm 0.35$ & $4.72 \pm 0.39$ & $4.10 \pm 0.57$ & 0.205 & Insignificant \\
\hline & & Right & $4.73 \pm 0.40$ & $4.40 \pm 0.38$ & $4.40 \pm 0.42$ & 0.069 & Insignificant \\
\hline & Rectus abdominis & Left & $4.63 \pm 0.60$ & $4.50 \pm 0.42$ & $4.50 \pm 0.00$ & 0.054 & Insignificant \\
\hline & & Right & $5.03 \pm 0.16$ & $5.08 \pm 0.30$ & $4.85 \pm 0.07$ & 0.048 & $A-C=0.049$ \\
\hline & Obliques & Left & $18.92 \pm 3.51$ & $17.75 \pm 3.18$ & $21.35 \pm 1.77$ & 0.780 & Insignificant \\
\hline & & Right & $18.18 \pm 2.83$ & $18.95 \pm 3.65$ & $17.35 \pm 7.00$ & 0.224 & Insignificant \\
\hline \multirow[t]{12}{*}{$6 \mathrm{mo}$} & Multifidus & Left & $4.25 \pm 0.23$ & $4.42 \pm 0.55$ & $4.15 \pm 0.07$ & 0.527 & Insignificant \\
\hline & & Right & $4.02 \pm 0.77$ & $4.06 \pm 0.51$ & $4.10 \pm 0.14$ & 0.259 & Insignificant \\
\hline & Erector spinae & Left & $9.16 \pm 0.65$ & $9.57 \pm 0.72$ & $10.25 \pm 0.92$ & 0.665 & Insignificant \\
\hline & & Right & $9.44 \pm 0.56$ & $10.17 \pm 1.00$ & $9.80 \pm 0.00$ & 0.043 & Insignificant \\
\hline & Ilio-psoas & Left & $6.50 \pm 0.34$ & $6.58 \pm 0.38$ & $6.70 \pm 0.14$ & 0.001 & $B-C=0.003, A-C=0.001$ \\
\hline & & Right & $5.84 \pm 0.54$ & $6.30 \pm 0.42$ & $6.25 \pm 0.50$ & 0.918 & Insignificant \\
\hline & Quadratus lumborum & Left & $4.33 \pm 0.56$ & $4.78 \pm 0.30$ & $4.45 \pm 0.07$ & 0.236 & Insignificant \\
\hline & & Right & $4.48 \pm 0.34$ & $4.59 \pm 0.29$ & $4.60 \pm 0.28$ & 0.348 & Insignificant \\
\hline & Rectus abdominis & Left & $4.81 \pm 0.40$ & $4.65 \pm 0.49$ & $4.85 \pm 0.78$ & 0.024 & $B-C=0.021, A-C=0.034$ \\
\hline & & Right & $5.13 \pm 0.16$ & $5.15 \pm 0.36$ & $5.50 \pm 0.00$ & 0.259 & Insignificant \\
\hline & Obliques & Left & $21.03 \pm 4.01$ & $20.37 \pm 3.02$ & $21.50 \pm 2.26$ & 0.697 & Insignificant \\
\hline & & Right & $21.80 \pm 2.16$ & $21.80 \pm 2.18$ & $24.65 \pm 0.50$ & 0.379 & Insignificant \\
\hline
\end{tabular}

Values are presented as mean \pm standard deviation. A-B means complete SCI versus incomplete SCl; B-C means incomplete SCl versus no deficit; A-C means complete $\mathrm{SCl}$ versus no deficit.

CSA, cross-sectional area; SCI, spinal cord injury; ASIA, American Spinal Injury Association.

${ }^{a}$ By one way analysis of variance test. 
Table 15. CSA \& fat content of lumbar muscles at L3-L4 disc level of spine according to initial neurological status in terms of complete and incomplete injury ( $n=51$ )

\begin{tabular}{|c|c|c|c|c|c|c|c|}
\hline \multirow[b]{2}{*}{ Duration } & \multirow{2}{*}{\multicolumn{2}{|c|}{$\begin{array}{l}\text { CSA }\left(\mathrm{cm}^{2}\right) \text { of lumbar } \\
\text { muscles (initially) }\end{array}$}} & \multicolumn{4}{|c|}{ Severity of initial injury } & \multirow[b]{2}{*}{$\begin{array}{l}\text { Intergroup } \\
\text { comparison }\end{array}$} \\
\hline & & & $\begin{array}{l}\text { Complete SCI } \\
\text { (ASIA grade A) } \\
(n=10)\end{array}$ & $\begin{array}{c}\text { Incomplete SCI } \\
\text { (ASIA grade } B, C, D) \\
(n=39)\end{array}$ & $\begin{array}{c}\text { No deficit } \\
\text { (ASIA grade E) } \\
(n=2)\end{array}$ & $p$-value ${ }^{\text {a) }}$ & \\
\hline \multirow[t]{12}{*}{ Initial } & Multifidus & Left & $4.67 \pm 0.42$ & $4.54 \pm 0.45$ & $4.40 \pm 0.28$ & 0.740 & Insignificant \\
\hline & & Right & $4.38 \pm 0.41$ & $4.30 \pm 0.43$ & $4.65 \pm 0.35$ & 0.411 & Insignificant \\
\hline & Erector Spinae & Left & $10.74 \pm 1.05$ & $12.74 \pm 3.30$ & $14.50 \pm 5.09$ & 0.308 & Insignificant \\
\hline & & Right & $9.82 \pm 0.71$ & $13.23 \pm 3.61$ & $14.70 \pm 5.09$ & 0.057 & Insignificant \\
\hline & Ilio-psoas & Left & $6.63 \pm 0.44$ & $6.65 \pm 0.39$ & $6.95 \pm 0.35$ & 0.273 & Insignificant \\
\hline & & Right & $6.35 \pm 0.50$ & $6.59 \pm 0.32$ & $6.55 \pm 0.50$ & 0.708 & Insignificant \\
\hline & Quadratus lumborum & Left & $4.81 \pm 0.23$ & $4.96 \pm 0.29$ & $4.65 \pm 0.07$ & 0.212 & Insignificant \\
\hline & & Right & $4.46 \pm 0.38$ & $4.80 \pm 0.31$ & $4.90 \pm 0.28$ & 0.267 & Insignificant \\
\hline & Rectus abdominis & Left & $4.95 \pm 0.43$ & $4.93 \pm 0.38$ & $5.10 \pm 0.00$ & 0.114 & Insignificant \\
\hline & & Right & $5.35 \pm 0.18$ & $5.39 \pm 0.37$ & $5.90 \pm 0.00$ & 0.281 & Insignificant \\
\hline & Obliques & Left & $21.42 \pm 3.26$ & $21.95 \pm 2.89$ & $22.50 \pm 1.70$ & 0.818 & Insignificant \\
\hline & & Right & $23.22 \pm 2.43$ & $22.76 \pm 3.17$ & $22.90 \pm 7.64$ & 0.264 & Insignificant \\
\hline \multirow[t]{12}{*}{$3 \mathrm{mo}$} & Multifidus & Left & $4.09 \pm 0.25$ & $4.09 \pm 0.28$ & $3.95 \pm 0.35$ & 0.277 & Insignificant \\
\hline & & Right & $3.87 \pm 0.23$ & $3.90 \pm 0.43$ & $3.95 \pm 0.21$ & 0.816 & Insignificant \\
\hline & Erector spinae & Left & $10.17 \pm 1.13$ & $12.77 \pm 3.87$ & $12.90 \pm 3.82$ & 0.103 & Insignificant \\
\hline & & Right & $9.34 \pm 0.55$ & $12.30 \pm 3.12$ & $13.00 \pm 3.82$ & 0.071 & Insignificant \\
\hline & Ilio-psoas & Left & $6.42 \pm 1.08$ & $6.37 \pm 0.59$ & $6.65 \pm 0.35$ & 0.260 & Insignificant \\
\hline & & Right & $5.99 \pm 0.53$ & $6.14 \pm 0.33$ & $6.20 \pm 0.28$ & 0.826 & Insignificant \\
\hline & Quadratus lumborum & Left & $4.63 \pm 0.32$ & $4.73 \pm 0.46$ & $3.95 \pm 0.35$ & 0.375 & Insignificant \\
\hline & & Right & $4.25 \pm 0.41$ & $4.34 \pm 0.43$ & $4.40 \pm 0.42$ & 0.315 & Insignificant \\
\hline & Rectus abdominis & Left & $4.73 \pm 0.54$ & $4.52 \pm 0.43$ & $4.50 \pm 0.00$ & 0.044 & $B-C=0.039$ \\
\hline & & Right & $4.98 \pm 0.23$ & $5.03 \pm 0.32$ & $4.85 \pm 0.07$ & 0.601 & Insignificant \\
\hline & Obliques & Left & $18.62 \pm 3.73$ & $18.37 \pm 2.96$ & $22.50 \pm 0.14$ & 0.449 & Insignificant \\
\hline & & Right & $18.85 \pm 2.82$ & $19.11 \pm 3.34$ & $18.85 \pm 6.58$ & 0.310 & Insignificant \\
\hline \multirow[t]{12}{*}{$6 \mathrm{mo}$} & Multifidus & Left & $4.19 \pm 0.27$ & $4.37 \pm 0.57$ & $4.15 \pm 0.07$ & 0.633 & Insignificant \\
\hline & & Right & $4.02 \pm 0.77$ & $4.06 \pm 0.51$ & $4.10 \pm 0.14$ & 0.259 & Insignificant \\
\hline & Erector spinae & Left & $9.72 \pm 0.95$ & $12.61 \pm 3.30$ & $13.75 \pm 4.45$ & 0.135 & Insignificant \\
\hline & & Right & $9.44 \pm 0.56$ & $12.32 \pm 2.95$ & $12.65 \pm 3.18$ & 0.072 & Insignificant \\
\hline & Ilio-psoas & Left & $6.43 \pm 0.36$ & $6.60 \pm 0.39$ & $6.70 \pm 0.14$ & 0.003 & $B-C=0.004, A-C=0.003$ \\
\hline & & Right & $5.92 \pm 0.53$ & $6.28 \pm 0.43$ & $6.20 \pm 0.42$ & 0.910 & Insignificant \\
\hline & Quadratus lumborum & Left & $4.33 \pm 0.56$ & $4.77 \pm 0.29$ & $4.45 \pm 0.07$ & 0.210 & Insignificant \\
\hline & & Right & $4.41 \pm 0.38$ & $4.58 \pm 0.31$ & $4.70 \pm 0.14$ & 0.540 & Insignificant \\
\hline & Rectus abdominis & Left & $4.80 \pm 0.41$ & $4.65 \pm 0.48$ & $4.85 \pm 0.78$ & 0.021 & $B-C=0.018, A-C=0.033$ \\
\hline & & Right & $5.13 \pm 0.16$ & $5.15 \pm 0.35$ & $5.50 \pm 0.00$ & 0.248 & Insignificant \\
\hline & Obliques & Left & $20.03 \pm 3.51$ & $19.82 \pm 4.00$ & $21.50 \pm 2.26$ & 0.415 & Insignificant \\
\hline & & Right & $22.35 \pm 2.05$ & $22.03 \pm 2.28$ & $21.45 \pm 4.03$ & 0.348 & Insignificant \\
\hline
\end{tabular}

Values are presented as mean \pm standard deviation. A-B means complete SCl versus incomplete SCI; B-C means incomplete SCI versus no deficit; A-C means complete SCl versus no deficit.

CSA, cross-sectional area; SCI, spinal cord injury; ASIA, American Spinal Injury Association.

${ }^{a}$ By one way analysis of variance test. 
Table 16. CSA \& fat content of lumbar muscles at L4-L5 disc level of spinal cord initially according to initial neurological status in terms of complete and incomplete injury ( $n=51)$

\begin{tabular}{|c|c|c|c|c|c|c|c|}
\hline \multirow[b]{2}{*}{ Duration } & \multirow[b]{2}{*}{$\begin{array}{c}\mathrm{CSA}\left(\mathrm{cm}^{2}\right) \text { of } \\
\text { lumbar muscles }\end{array}$} & & \multicolumn{4}{|c|}{ Severity of initial injury } & \multirow[b]{2}{*}{$\begin{array}{l}\text { Intergroup } \\
\text { comparison }\end{array}$} \\
\hline & & & $\begin{array}{c}\text { Complete SCI } \\
\text { (ASIA grade A) } \\
(n=10)\end{array}$ & $\begin{array}{c}\text { Incomplete SCI } \\
\text { (ASIA grade B, C, D) } \\
(n=39)\end{array}$ & $\begin{array}{c}\text { No deficit } \\
\text { (ASIA grade E) } \\
(n=2)\end{array}$ & $p$-value ${ }^{\text {a) }}$ & \\
\hline \multirow[t]{12}{*}{ Initial } & Multifidus & Left & $8.31 \pm 0.66$ & $7.12 \pm 1.38$ & $9.35 \pm 1.34$ & 0.130 & Insignificant \\
\hline & & Right & $7.31 \pm 0.84$ & $7.00 \pm 1.50$ & $8.70 \pm 1.27$ & 0.690 & Insignificant \\
\hline & Erector spinae & Left & $10.36 \pm 0.86$ & $9.66 \pm 0.48$ & $9.60 \pm 0.00$ & 0.250 & Insignificant \\
\hline & & Right & $9.82 \pm 0.71$ & $9.69 \pm 0.49$ & $9.85 \pm 0.35$ & 0.470 & Insignificant \\
\hline & Ilio-psoas & Left & $6.63 \pm 0.44$ & $6.65 \pm 0.39$ & $6.70 \pm 0.00$ & 0.287 & Insignificant \\
\hline & & Right & $6.34 \pm 0.50$ & $6.59 \pm 0.31$ & $6.55 \pm 0.50$ & 0.465 & Insignificant \\
\hline & Quadratus lumborum & Left & $4.81 \pm 0.29$ & $4.98 \pm 0.30$ & $4.65 \pm 0.07$ & 0.192 & Insignificant \\
\hline & & Right & $4.50 \pm 0.39$ & $4.81 \pm 0.30$ & $4.90 \pm 0.28$ & 0.260 & Insignificant \\
\hline & Rectus abdominis & Left & $5.05 \pm 0.38$ & $4.92 \pm 0.38$ & $5.10 \pm 0.00$ & 0.129 & Insignificant \\
\hline & & Right & $5.35 \pm 0.18$ & $5.35 \pm 0.42$ & $5.90 \pm 0.00$ & 0.179 & Insignificant \\
\hline & Obliques & Left & $21.47 \pm 3.30$ & $22.40 \pm 2.81$ & $22.50 \pm 1.70$ & 0.496 & Insignificant \\
\hline & & Right & $22.66 \pm 1.22$ & $23.26 \pm 2.81$ & $24.80 \pm 4.95$ & 0.258 & Insignificant \\
\hline \multirow[t]{12}{*}{$3 \mathrm{mo}$} & Multifidus & Left & $6.40 \pm 0.37$ & $5.92 \pm 1.17$ & $8.05 \pm 1.63$ & 0.251 & Insignificant \\
\hline & & Right & $6.50 \pm 0.37$ & $6.20 \pm 1.38$ & $7.50 \pm 1.70$ & 0.538 & Insignificant \\
\hline & Erector spinae & Left & $9.44 \pm 0.47$ & $9.22 \pm 0.58$ & $9.50 \pm 0.00$ & 0.434 & Insignificant \\
\hline & & Right & $9.29 \pm 0.57$ & $9.32 \pm 0.50$ & $9.45 \pm 0.21$ & 0.525 & Insignificant \\
\hline & Ilio-psoas & Left & $6.52 \pm 0.72$ & $6.43 \pm 0.58$ & $6.95 \pm 0.78$ & 0.214 & Insignificant \\
\hline & & Right & $5.97 \pm 0.55$ & $6.14 \pm 0.31$ & $6.20 \pm 0.28$ & 0.691 & Insignificant \\
\hline & Quadratus lumborum & Left & $4.49 \pm 0.33$ & $4.71 \pm 0.46$ & $4.10 \pm 0.57$ & 0.147 & Insignificant \\
\hline & & Right & $4.93 \pm 0.32$ & $4.37 \pm 0.42$ & $4.40 \pm 0.42$ & 0.062 & Insignificant \\
\hline & Rectus abdominis & Left & $4.72 \pm 0.55$ & $4.48 \pm 0.42$ & $4.50 \pm 0.00$ & 0.051 & Insignificant \\
\hline & & Right & $5.03 \pm 0.16$ & $5.06 \pm 0.33$ & $4.85 \pm 0.07$ & 0.021 & Insignificant \\
\hline & Obliques & Left & $19.68 \pm 2.72$ & $19.20 \pm 2.69$ & $22.85 \pm 2.19$ & 0.913 & Insignificant \\
\hline & & Right & $19.50 \pm 2.32$ & $19.86 \pm 4.09$ & $13.35 \pm 5.73$ & 0.485 & Insignificant \\
\hline \multirow[t]{12}{*}{$6 \mathrm{mo}$} & Multifidus & Left & $8.47 \pm 0.69$ & $6.97 \pm 1.38$ & $9.05 \pm 0.64$ & 0.042 & Insignificant \\
\hline & & Right & $8.40 \pm 0.73$ & $6.61 \pm 1.34$ & $7.60 \pm 1.41$ & 0.084 & Insignificant \\
\hline & Erector spinae & Left & $9.16 \pm 0.65$ & $9.43 \pm 0.47$ & $9.60 \pm 0.00$ & 0.707 & Insignificant \\
\hline & & Right & $9.42 \pm 0.57$ & $9.53 \pm 0.50$ & $9.25 \pm 0.78$ & 0.599 & Insignificant \\
\hline & Ilio-psoas & Left & $6.51 \pm 0.33$ & $6.60 \pm 0.40$ & $6.70 \pm 0.00$ & 0.005 & $B-C=0.006, A-C=0.004$ \\
\hline & & Right & $5.97 \pm 0.53$ & $6.26 \pm 0.32$ & $6.25 \pm 0.50$ & 0.487 & Insignificant \\
\hline & Quadratus lumborum & Left & $4.25 \pm 0.60$ & $4.78 \pm 0.29$ & $4.45 \pm 0.07$ & 0.214 & Insignificant \\
\hline & & Right & $4.36 \pm 0.41$ & $4.56 \pm 0.31$ & $4.60 \pm 0.28$ & 0.599 & Insignificant \\
\hline & Rectus abdominis & Left & $4.81 \pm 0.40$ & $4.63 \pm 0.48$ & $4.85 \pm 0.78$ & 0.020 & $B-C=0.016, A-C=0.044$ \\
\hline & & Right & $5.13 \pm 0.16$ & $5.16 \pm 0.37$ & $5.50 \pm 0.00$ & 0.139 & Insignificant \\
\hline & Obliques & Left & $20.01 \pm 3.27$ & $19.86 \pm 3.66$ & $21.50 \pm 2.26$ & 0.297 & Insignificant \\
\hline & & Right & $20.50 \pm 1.96$ & $21.29 \pm 2.82$ & $24.65 \pm 0.50$ & 0.274 & Insignificant \\
\hline
\end{tabular}

Values are presented as mean \pm standard deviation. A-B means complete SCI versus incomplete SCl; B-C means incomplete SCI versus no deficit; A-C means complete SCl versus no deficit.

CSA, cross-sectional area; SCl, spinal cord injury; ASIA, American Spinal Injury Association.

${ }^{a}$ By one way analysis of variance test. 
Table 17. CSA \& fat content of lumbar muscles at L5-S1 disc level of spinal cord initially according to neurological status in terms of complete and incomplete injury $(n=51)$

\begin{tabular}{|c|c|c|c|c|c|c|c|}
\hline \multirow[b]{2}{*}{ Duration } & \multirow{2}{*}{\multicolumn{2}{|c|}{$\begin{array}{l}\text { CSA }\left(\mathrm{cm}^{2}\right) \text { of } \\
\text { lumber muscles }\end{array}$}} & \multicolumn{4}{|c|}{ Severity of initial injury } & \multirow[b]{2}{*}{$\begin{array}{l}\text { Intergroup } \\
\text { comparison }\end{array}$} \\
\hline & & & $\begin{array}{c}\text { Complete SCI } \\
\text { (ASIA grade A) } \\
(n=10)\end{array}$ & $\begin{array}{c}\text { Incomplete SCI } \\
\text { (ASIA grade B, C, D) } \\
(\mathrm{n}=39)\end{array}$ & $\begin{array}{c}\text { No deficit } \\
\text { (ASIA grade E) } \\
(n=2)\end{array}$ & $p$-value ${ }^{\text {a) }}$ & \\
\hline \multirow[t]{8}{*}{ Initial } & Multifidus & Left & $9.41 \pm 1.43$ & $9.40 \pm 0.82$ & $9.10 \pm 0.85$ & 0.885 & Insignificant \\
\hline & & Right & $9.43 \pm 0.98$ & $9.56 \pm 0.90$ & $9.40 \pm 0.85$ & 0.441 & Insignificant \\
\hline & Erector spinae & Left & $10.36 \pm 0.86$ & $9.66 \pm 0.48$ & $9.60 \pm 0.00$ & 0.212 & Insignificant \\
\hline & & Right & $9.82 \pm 0.71$ & $9.65 \pm 0.48$ & $9.85 \pm 0.35$ & 0.481 & Insignificant \\
\hline & Ilio-psoas & Left & $16.87 \pm 2.51$ & $16.25 \pm 0.58$ & $16.25 \pm 0.35$ & 0.989 & Insignificant \\
\hline & & Right & $16.96 \pm 2.07$ & $14.15 \pm 1.45$ & $13.25 \pm 1.63$ & 0.191 & Insignificant \\
\hline & Rectus abdominis & Left & $4.95 \pm 0.43$ & $4.93 \pm 0.37$ & $5.10 \pm 0.00$ & 0.107 & Insignificant \\
\hline & & Right & $5.35 \pm 0.18$ & $5.36 \pm 0.41$ & $5.90 \pm 0.00$ & 0.130 & Insignificant \\
\hline \multirow[t]{8}{*}{$3 \mathrm{mo}$} & Multifidus & Left & $8.70 \pm 1.17$ & $9.07 \pm 0.77$ & $9.20 \pm 0.99$ & 0.466 & Insignificant \\
\hline & & Right & $8.72 \pm 1.42$ & $8.99 \pm 1.25$ & $8.00 \pm 0.00$ & 0.881 & Insignificant \\
\hline & Erector spinae & Left & $9.46 \pm 0.58$ & $9.38 \pm 0.60$ & $9.50 \pm 0.00$ & 0.200 & Insignificant \\
\hline & & Right & $9.34 \pm 0.55$ & $9.23 \pm 0.62$ & $9.45 \pm 0.21$ & 0.037 & $A-B=0.032$ \\
\hline & Ilio-psoas & Left & $15.92 \pm 2.15$ & $14.51 \pm 0.46$ & $14.40 \pm 0.28$ & 0.437 & Insignificant \\
\hline & & Right & $15.76 \pm 1.81$ & $12.84 \pm 1.87$ & $12.85 \pm 1.91$ & 0.461 & Insignificant \\
\hline & Rectus abdominis & Left & $4.72 \pm 0.55$ & $4.49 \pm 0.44$ & $4.50 \pm 0.00$ & 0.065 & Insignificant \\
\hline & & Right & $4.96 \pm 0.14$ & $5.02 \pm 0.35$ & $4.85 \pm 0.07$ & 0.019 & $A-C=0.025$ \\
\hline \multirow[t]{8}{*}{$6 \mathrm{mo}$} & Multifidus & Left & $8.87 \pm 1.70$ & $9.19 \pm 0.69$ & $9.40 \pm 0.42$ & 0.649 & Insignificant \\
\hline & & Right & $9.01 \pm 1.02$ & $9.08 \pm 1.04$ & $8.85 \pm 0.92$ & 0.240 & Insignificant \\
\hline & Erector spinae & Left & $9.40 \pm 0.33$ & $9.43 \pm 0.46$ & $9.60 \pm 0.00$ & 0.677 & Insignificant \\
\hline & & Right & $9.39 \pm 0.48$ & $9.55 \pm 0.60$ & $9.25 \pm 0.78$ & 0.666 & Insignificant \\
\hline & Ilio-psoas & Left & $16.18 \pm 2.17$ & $14.39 \pm 1.06$ & $14.70 \pm 0.28$ & 0.834 & Insignificant \\
\hline & & Right & $16.04 \pm 1.49$ & $13.66 \pm 1.42$ & $13.15 \pm 1.77$ & 0.164 & Insignificant \\
\hline & Rectus abdominis & Left & $4.81 \pm 0.40$ & $4.65 \pm 0.48$ & $4.85 \pm 0.78$ & 0.021 & $B-C=0.017, A-C=0.032$ \\
\hline & & Right & $5.13 \pm 0.16$ & $5.14 \pm 0.36$ & $5.50 \pm 0.00$ & 0.277 & Insignificant \\
\hline
\end{tabular}

Values are presented as mean \pm standard deviation. A-B means complete SCl versus incomplete SCI; B-C means incomplete SCI versus no deficit; A-C means complete $\mathrm{SCl}$ versus no deficit.

CSA, cross-sectional area; SCI, spinal cord injury; ASIA, American Spinal Injury Association.

${ }^{a} B y$ one way analysis of variance test.

disease [12]. Postoperative computed tomography (CT) and MRI imaging studies have shown that reductions in the CSA of the spinal muscles occur, as well as changes in muscular density. Gille et al. [16] reported that erector spinae muscle alterations mainly occur at locations distal to the posterior lumbar surgical procedures. Kim et al. [17] observed a significant decrease in the CSA of the multifidus muscle in patients who underwent open pedicle fixation. Fan et al. $[10,18]$ reported that multifidus atrophy was reduced among patients who underwent minimally invasive treatment $(p<0.001)$, with mean reductions in the
CSA of $12.2 \%$ at the operative level and $8.5 \%$ at the adjacent levels compared with $36.8 \%$ and $29.3 \%$, respectively, among patients who underwent conventional open surgery. Conversely, Keller et al. [19] observed that there was no decrease in spinal muscle CSA after surgery, but muscle density (determined by CT) was adversely affected.

The abovementioned studies indicate that surgical intervention with pedicle screws for the traumatic or degenerative disorders of the spine can lead to alteration in the CSA and fat contents of the paravertebral muscles [10,16-18]. In the present study, we were unable to clarify 
whether operative intervention with pedicle screws causes further damage to paravertebral muscles because no significant differences were observed in the CSAs of various muscles (except at the L1-L2 level, which was adjacent to the most commonly injured site, for the erector spinae and rectus abdominis) between groups divided according to conservative or operative management. However, all patients in this study underwent open pedicle screw fixation. Ntilikina et al. [20] compared the MRI findings of paravertebral muscles after implant removal in thoracolumbar fractures after open versus percutaneous instrumentation. They reported that percutaneous instrumentation led to decreased muscle atrophy compared with open surgery. The MRI signal differences for T12 and L1 fractures suggested reduced fat infiltration within the CSA of patients who received percutaneous treatment. A major difference between the study of Ntilikina et al. [20] and the present study was that none of the patients in the former had neurological deficits. Similarly, other studies (such as those of Gille et al. [16], Kim et al. [17], and Fan et al. $[10,18])$ reported differences in the pre- and postoperative CSA of muscles after lumbar spine surgery on patients without major neurological deficits. Anecdotal evidence from the literature suggests that further damage should be expected after open pedicle screw in previously paralyzed muscles [10,13,15-17].

The degree of neurological involvement has a significant effect on the wastage of the paravertebral muscle post-SCI [2]. We did not find any statistically significant differences between patients categorized as motor complete (ASIA A) and motor incomplete (ASIA B, C, or D) in the present study, and intergroup comparisons at different time points did not reveal any significant changes in the majority of the muscles studied. To the best of our knowledge, no study has reported such findings. This could be due to the short follow-up of the present study (6 months) and our robust post-SCI rehabilitation protocol irrespective of the degree of neurological involvement. Furthermore, the lack of significant differences could have been influenced by the small number of patients with complete SCI in the present cohort. Larger studies involving more patients and longer follow-up are required to verify these findings.

\section{Conclusions}

Spinal trauma leads to alterations in the vertebral column, spinal cord, intervertebral discs, and paraspinal muscle morphology in the initial phase after injury. The extent of these changes may determine the initial neurological deficit and subsequent recovery. Although the present study did not identify any statistically significant effects of neurological status or management strategy on these parameters, improvement in the later phase of recovery was observed in response to rehabilitation. Future studies are needed to evaluate the exact mechanisms underlying these alterations and the benefits of rehabilitation strategies with regard to these parameters.

\section{Conflict of Interest}

No potential conflict of interest relevant to this article was reported.

\section{References}

1. Comarr AE, Hutchinson RH, Bors E. Extremity fractures of patients with spinal cord injuries. Am J Surg 1962;103:732-9.

2. Singh R, Rohilla RK, Saini G, Kaur K. Longitudinal study of body composition in spinal cord injury patients. Indian J Orthop 2014;48:168-77.

3. Verga S, Buscemi S, Caimi G. Resting energy expenditure and body composition in morbidly obese, obese and control subjects. Acta Diabetol 1994;31:4751.

4. Singh R, Yadav SK, Sood S, Yadav RK, Rohilla R. Magnetic resonance imaging of lumbar trunk parameters in chronic low backache patients and healthy population: a comparative study. Eur Spine J 2016;25:2864-72.

5. Harvey LA. Physiotherapy rehabilitation for people with spinal cord injuries. J Physiother 2016;62:4-11.

6. Chafetz RS, Vogel LC, Betz RR, Gaughan JP, Mulcahey MJ. International standards for neurological classification of spinal cord injury: training effect on accurate classification. J Spinal Cord Med 2008;31:53842.

7. McCormack T, Karaikovic E, Gaines RW. The load sharing classification of spine fractures. Spine (Phila Pa 1976) 1994;19:1741-4.

8. Vaccaro AR, Zeiller SC, Hulbert RJ, et al. The thoracolumbar injury severity score: a proposed treatment algorithm. J Spinal Disord Tech 2005;18:209-15.

9. Arija-Blazquez A, Ceruelo-Abajo S, Diaz-Merino 
MS, et al. Effects of electromyostimulation on muscle and bone in men with acute traumatic spinal cord injury: a randomized clinical trial. J Spinal Cord Med 2014;37:299-309.

10. Fan S, Hu Z, Zhao F, Zhao X, Huang Y, Fang X. Multifidus muscle changes and clinical effects of onelevel posterior lumbar interbody fusion: minimally invasive procedure versus conventional open approach. Eur Spine J 2010;19:316-24.

11. O’leary S, Jull G, Van Wyk L, Pedler A, Elliott J. Morphological changes in the cervical muscles of women with chronic whiplash can be modified with exercise: a pilot study. Muscle Nerve 2015;52:772-9.

12. Cha JR, Kim YC, Yoon WK, et al. The recovery of damaged paraspinal muscles by posterior surgical treatment for patients with lumbar degenerative diseases and its clinical consequence. J Back Musculoskelet Rehabil 2017;30:801-9.

13. Hicks AL, Martin KA, Ditor DS, et al. Long-term exercise training in persons with spinal cord injury: effects on strength, arm ergometry performance and psychological well-being. Spinal Cord 2003;41:34-43.

14. Mulroy SJ, Thompson L, Kemp B, et al. Strengthening and optimal movements for painful shoulders (STOMPS) in chronic spinal cord injury: a randomized controlled trial. Phys Ther 2011;91:305-24.
15. Ditunno JF Jr, Cohen ME, Hauck WW, Jackson AB, Sipski ML. Recovery of upper-extremity strength in complete and incomplete tetraplegia: a multicenter study. Arch Phys Med Rehabil 2000;81:389-93.

16. Gille O, Jolivet E, Dousset V, et al. Erector spinae muscle changes on magnetic resonance imaging following lumbar surgery through a posterior approach. Spine (Phila Pa 1976) 2007;32:1236-41.

17. Kim DY, Lee SH, Chung SK, Lee HY. Comparison of multifidus muscle atrophy and trunk extension muscle strength: percutaneous versus open pedicle screw fixation. Spine (Phila Pa 1976) 2005;30:123-9.

18. Fan SW, Hu ZJ, Fang XQ, Zhao FD, Huang Y, Yu $\mathrm{HJ}$. Comparison of paraspinal muscle injury in onelevel lumbar posterior inter-body fusion: modified minimally invasive and traditional open approaches. Orthop Surg 2010;2:194-200.

19. Keller A, Gunderson R, Reikeras O, Brox JI. Reliability of computed tomography measurements of paraspinal muscle cross-sectional area and density in patients with chronic low back pain. Spine (Phila Pa 1976) 2003;28:1455-60.

20. Ntilikina Y, Bahlau D, Garnon J, et al. Open versus percutaneous instrumentation in thoracolumbar fractures: magnetic resonance imaging comparison of paravertebral muscles after implant removal. J Neurosurg Spine 2017;27:235-41. 